\title{
Machine Learning Optimization for Prostate Brachytherapy Treatment Planning
}

by

Alexandru M. Nicolae, BSc (Hon), RTT

University of Toronto, Toronto, ON, Canada

\author{
A thesis \\ presented to Ryerson University \\ in partial fulfillment of the degree of \\ Master of Science \\ in the graduate program of \\ Biomedical Physics
}

(C)July 2016, Alexandru M. Nicolae

Toronto, ONN, Canada 


\section{Author's Declaration}

I hereby declare that I am the sole author of this thesis. This is a true copy of the thesis including any required final revisions, as accepted by my examiners.

I authorize Ryerson University to lend this thesis or dissertation to other institutions or individuals for the purpose of scholarly research.

I further authorize Ryerson University to reproduce this thesis by photocopying or by other means, in total or in part, at the request of other institutions or individuals for the purpose of scholarly research.

I understand that my thesis may be made electronically available to the public. 


\begin{abstract}
Machine Learning Optimization for Prostate Brachytherapy Treatment Planning

\author{
Alexandru Nicolae \\ Master of Science \\ Ryerson University
}

2016

Prostate Low-Dose-Rate brachytherapy (LDR) is one of the most effective treatments for localized prostate cancer. Machine Learning (ML), the application of statistics to complex computational problem solving, was applied to prostate LDR brachytherapy treatment planning. Planning time, pre-implant dosimetry, and various measures of clinical implant quality for ML plans were compared against plans created by expert brachytherapists.
\end{abstract}

The average planning time to create an ML plan was $0.84 \pm 0.57$ min compared to over $17.88 \pm 8.76 \mathrm{~min}$ for an experienced brachytherapists. Dosimetry was not significantly different for ML and expert brachytherapist plans. Clinical implant quality for the ML plans was ranked as nearly equivalent to the brachytherapist treatment plans in all qualitative categories evaluated.

The results of this thesis demonstrate that it is possible to generate high quality prostate brachytherapy treatment plans with comparable quality to those of a human expert using a custom ML algorithm. 


\section{Acknowledgements}

I am very grateful and thankful to my supervisor Ananth Ravi for his support, encouragement and mentorship throughout the years. Dr. Ravi has been an instrumental part in the development of my skills from a simple radiation therapist to that of a highly trained and knowledgeable researcher.

I would like to also thank Emily Heath for training me in the finer points of computer optimization as an eager and ambitious undergraduate student. Thank you for your continued support throughout these years.

I would like to thank my committee members including Carl Kumaradas, and Raffi Karshafian for their much needed and very helpful feedback on our work, and for their guidance in navigating the complex graduate student journey.

I would like to give a warm thanks to our many clinical collaborators (both national and international) who provided their much needed expertise to evaluate this project. Many thanks to Dr. Gerard Morton, Dr. Hans Chung, Dr. Andrew Loblaw, Dr. Suneil Jain, Dr. Darren Mitchell, Dr. Joelle Helou, Dr. Motasem Al-Hanaqta, and Lin Lu.

Finally, I am very grateful for the support my mother, father, and brother have given me throughout this time. Despite not always fully understanding what I do every day your support helped me get through this challenging time in my life. 


\section{Contents}

Authors Declaration $\quad$ i

Abstract $\quad$ iii

Acknowledgements $\quad$ iv

1 Introduction 1

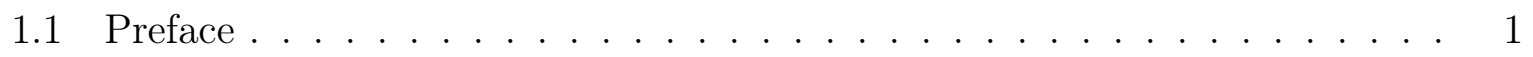

1.2 Prostate Cancer . . . . . . . . . . . . . . . . . . . . . . . . 1

1.2.1 Epidemiology \& Etiology . . . . . . . . . . . . . . . . . . 1

1.2 .2 Increased Incidence . . . . . . . . . . . . . . . . . . 2

1.2.3 Anatomy \& Physiology . . . . . . . . . . . . . . . . . . . 2

1.2.4 Pathophysiology . . . . . . . . . . . . . . . . 3

1.2.5 Prostate Cancer Screening . . . . . . . . . . . . . . . . 3

1.2.6 Diagnosis \& Staging . . . . . . . . . . . . . . 5

1.3 Management of Localized Prostate Cancer . . . . . . . . . . . . . . 6

1.3.1 Active Surveillance . . . . . . . . . . . . . . . . 6 
1.3.2 Active Treatment . . . . . . . . . . . . . . . . 8

1.4 Current Issues in Brachytherapy . . . . . . . . . . . . . . . . . . . . . . 11

1.4.1 The Low-Dose-Rate Workflow . . . . . . . . . . . . . . . . . . . 11

1.4.2 Limitations of Treatment Planning Methodologies . . . . . . . . . . 13

1.5 Potential Solutions to the Planning Problem . . . . . . . . . . . . . . 15

1.5.1 Overview of Strategy to Be Implemented . . . . . . . . . . . . . . . 15

1.5.2 Stochastic Optimization . . . . . . . . . . . . . . 16

1.5.3 Deficiencies of Naive Stochastic Optimization . . . . . . . . . . . . 19

1.5.4 Incorporation of Machine Learning . . . . . . . . . . . . . . . 20

1.6 Hypothesis . . . . . . . . . . . . . . . . . . . . . . . 22

1.7 Potential Impact . . . . . . . . . . . . . . . . . . . . . . . 22

1.8 Thesis Overview . . . . . . . . . . . . . . . . . . . . . . 23

2 Evaluation of a Machine Learning Algorithm for Prostate LDR Brachytherapy

2.1 Abstract . . . . . . . . . . . . . . . . . . . 24

2.2 Introduction . . . . . . . . . . . . . . . . . . 25

2.3 Materials \& Methods . . . . . . . . . . . . . . . . . . . . 27

2.3.1 Data Description . . . . . . . . . . . . . . . 27

2.3.2 System Architecture . . . . . . . . . . . . . . . . . . . 27

2.3.3 Study Design . . . . . . . . . . . . . . . . . . . 32

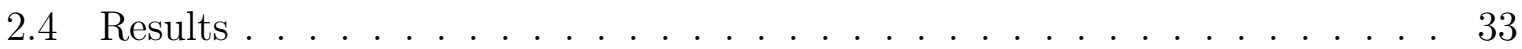


2.5 Discussion . . . . . . . . . . . . . . . . . . 37

2.6 Conclusion . . . . . . . . . . . . . . . . . . . . . . . 39

$\begin{array}{lll}3 & \text { Conclusion } & 40\end{array}$

3.1 Thesis Summary . . . . . . . . . . . . . . . . . . . . . 40

3.2 Discussion \& Future Work . . . . . . . . . . . . . . . . . . . . . . . 42

3.2.1 Further Optimization of the ML algorithm . . . . . . . . . . . . 42

3.2.2 Machine Learning for HDR Brachytherapy . . . . . . . . . . . . . . 45

3.2.3 Machine Learning for Prostate Radiotherapy . . . . . . . . . . . . . 46

Bibliography 46 


\section{List of Tables}

1.1 NCCN risk groups for prostate cancer . . . . . . . . . . . . 7

2.1 Clinical rule strategies for constraining optimization . . . . . . . . . . 31

2.2 ABS dose criteria for treatment plan evaluation . . . . . . . . . . . 32 


\section{List of Figures}

1.1 Anatomy of the Prostate . . . . . . . . . . . . . . 4

1.2 Zones of the Prostate . . . . . . . . . . . . . . . 5

1.3 Prostate cancer control rates for different treatment modalities . . . . . . . 9

1.4 LDR brachytherapy . . . . . . . . . . . . . . . . . . . 12

1.5 Source for brachytherapy dose calculations . . . . . . . . . . . . . 17

1.6 Prostate surface dose computation example . . . . . . . . . . . . . . 18

1.7 Optimized Inverse Planned Simulated Annealing plan . . . . . . . . . . . . 20

2.1 Machine-Learning workflow for LDR brachytherapy . . . . . . . . . . . 28

2.2 Results of prospective planning time comparison . . . . . . . . . . . . 33

2.3 Results of Pre-implant plan dosimetry comparison between groups . . . . . 34

2.4 Results of expert clinical qualitative study . . . . . . . . . . . . 35

2.5 Results demonstrating isodose treatment plan comparisons between groups 36 


\section{List of Abbreviations}

ABS American Brachytherapy Society. 11

ACS American Cancer Society. 4, 11

AJCC American Joint Commission on Cancer. 5

AS Active Surveillance. 6

AUA American Urological Association. 11

BPH Benign Prostatic Hyperplasia. 3

BT Brachytherapy. 10, 11

CT Computed Tomography. 6

DICOM Digital Imaging and Communications in Medicine. 28

DVH Dose-Volume Histogram. 13

EBRT External Beam Radiation Therapy. 8

ERSPC European Randomized Study of Screening for Prostate Cancer. 4

GS Gleason Score. 5

HDR High-Dose-Rate brachytherapy. 10

HIPO Hybrid Inverse Planning and Optimization. 15 
I-125 Iodine-125. 10

IPSA Inverse Planned Simulated Annealing. 15

Ir-192 Iridium-192. 10

LDR Low-Dose-Rate brachytherapy. iii

MI Mutual Information. 21

ML Machine Learning. iii

MRI Magnetic Resonance Imaging. 6

NCCN National Comprehensive Cancer Network. 4

OAR Organs-at-Risk. 11

OR Odds Ratio. 35

PCA Principle Component Analysis. 21

PLCO Prostate, Lung, Colorectal, Ovarian screening trial. 4

PSA Prostate-Specific Antigen. 3

RP Radical Prostatectomy. 8

RT Radiation Therapist / Dosimetrist. 12, 26

TRUS Trans-Rectal Ultrasound. 6

WW Watchful Waiting. 7 


\section{Chapter 1}

\section{Introduction}

\subsection{Preface}

Chapter 2 of this thesis represents an under-review manuscript: "Alexandru-Mihai Nicolae, Gerard Morton, Hans Chung, Andrew Loblaw, Suneil Jain, Darren Mitchell, Lin Lu, Joelle Helou, Motasem Al-Hanaqta, Emily Heath, Ananth Ravi. Evaluation of a novel machine learning algorithm for treatment planning in low-dose-rate (LDR) prostate brachytherapy" submitted to the International Journal of Radiation Oncology Biology and Physics. Nicolae AM was responsible for drafting the manuscript, collection and administration of the research studies, and analysis of data.

\subsection{Prostate Cancer}

\subsubsection{Epidemiology \& Etiology}

In recent years cancer has become the leading cause of mortality among Canadians, surpassing cardiovascular disease [1]. Improvements in cancer survival in the past decade have largely been due to comprehensive screening programs, as well as improvements in cancer therapies. The most common form of cancer in Canadian men is prostate cancer, it also accounts for over $10 \%$ of all cancer-related deaths. Over 24,000 men will be diagnosed 
with prostate cancer in 2016 with over 4,000 dying from the disease making it a significant health care burden. Due to the high incidence rate and an average five-year survival rate of $96 \%$ prostate cancer has a high prevalence among the Canadian population, as high as $14 \%[2]$.

Several factors place men at an increased risk of developing prostate cancer. Family history of the disease [3], poor diet or unhealthy weight [4], and race / ethnicity (men of African descent are at increased risk of developing the disease, while men of Asian descent are at a decreased risk) [5]. The most dominant risk factor for developing prostate cancer however is advanced age. Men over 50 years of age are far more likely to develop prostate cancer. Approximately $60 \%$ of men diagnosed with prostate cancer are over the age of 65 with the median age at diagnosis being 66 years [6].

\subsubsection{Increased Incidence}

By 2030 it is predicted that the incidence of prostate cancer will increase by $97 \%$, a near doubling from the current annual incidence rate [2]. This extrapolated increase is largely the result of the aging post-World War II baby boomer population. Approximately $63 \%$ of all prostate cancers are diagnosed at a stage where the cancer is confined to the gland (localized disease); this has been attributed to early screening programs to detect the cancer in otherwise asymptomatic men [7]. The large increase in the cohort of patients with controllable forms of prostate cancer will place a significant burden on Canada's already strained healthcare resources. One method of scaling current treatment programs to account for these dramatic increases in incidence is to redesign current treatment approaches favoring effective and resource-efficient treatment alternatives.

\subsubsection{Anatomy \& Physiology}

The prostate gland is a small exocrine gland situated in the male genitourinary system inferior to the neck of the urinary bladder; the urethra passes directly through the gland and the rectum sits posterior to the gland (Fig 1.1). The gland functions in maintaining normal reproductive health by furnishing nutrients, enzymes, and proteins critical for the 
functioning of sperm. The anatomy of the gland itself is commonly divided into several zones (Fig 1.2) [8]. One of the key secretory proteins excreted by the epithelial cells of the prostate gland is Prostate-Specific Antigen (PSA), a glycoprotein that helps ensure sperm motility. Serum PSA, the amount of PSA circulating within the blood stream, may be slightly elevated in even healthy patients or several non-cancerous prostate conditions, such as Benign Prostatic Hyperplasia (BPH). In patients with prostate cancer the level of serum PSA may be significantly elevated [8 - 10].

\subsubsection{Pathophysiology}

Prostate cancer, or adenocarcinoma of the prostate, occurs when the glandular cells that make up the prostate gland no longer respond to normal growth signals and begin to divide uncontrollably. More locally advanced cancers will outgrow the prostatic capsule itself and infiltrate nearby organs such as the urinary bladder and rectum. Very aggressive cancers may additionally infiltrate the lymphatic system and spread to other more distant organs, a process known as metastasis. Most commonly advanced prostate cancers may spread to bones in the pelvis or extremities [11]. Because of the proximity of some of these cancers to the urethra and urinary bladder they may produce urinary symptoms in patients; however, prostate cancer may be largely asymptomatic in the majority of patients, screening programs are generally required for early detection of the disease.

\subsubsection{Prostate Cancer Screening}

Although largely asymptomatic, early detection of localized prostate cancer is often accomplished through early screening programs. These screening programs rely on measurement of serum PSA in patients with known risk factors for the disease, namely advanced age, but who otherwise may be asymptomatic. Early disease screening in the general population has allowed stage migration towards more favorable disease; stage migration refers to the detection of prostate cancer at earlier stages where treatment interventions are more likely to be effective [12-14]. Despite the benefits of early detection there has been considerable debate as to whether PSA screening in asymptomatic patients may 


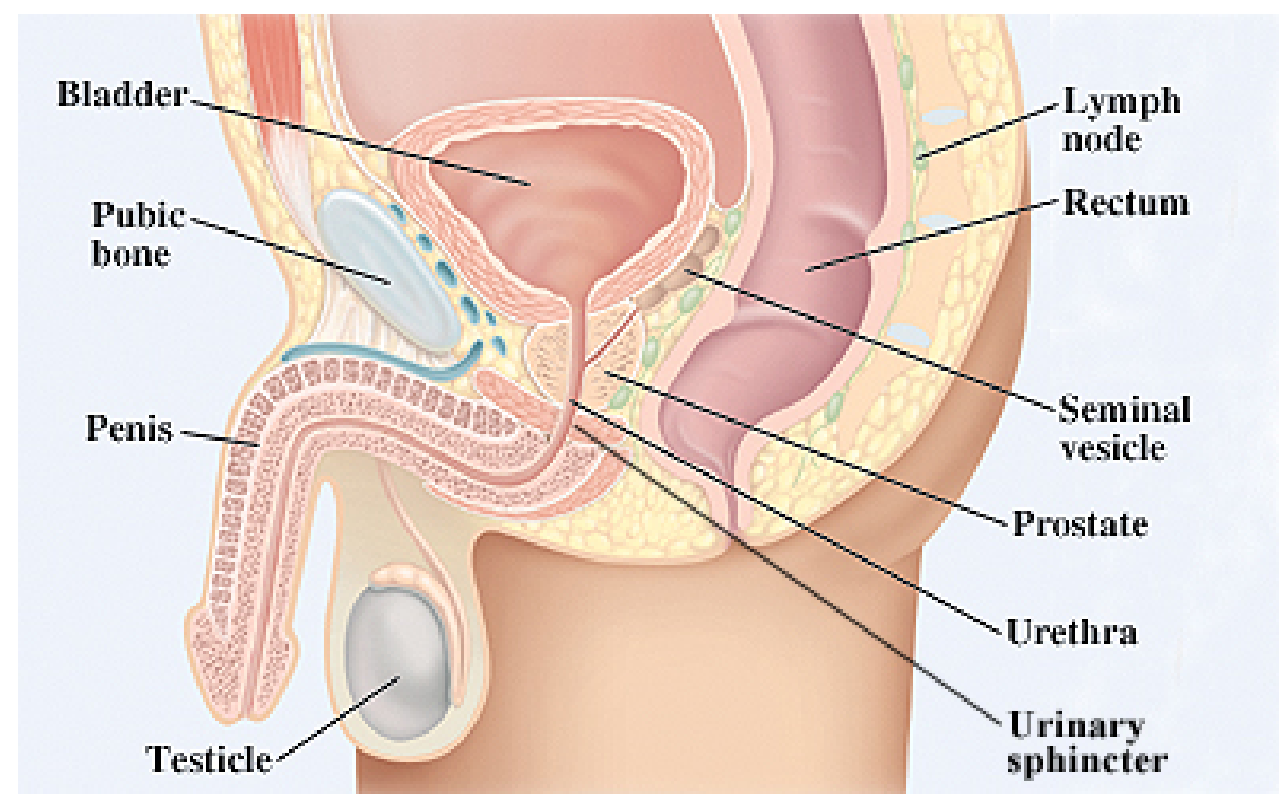

Figure 1.1: Anatomy of the prostate and surrounding organs-at-risk. Ref: http://www.fairview.org/healthlibrary/Article/87095.

play a role in the over-diagnosis and over-treatment of prostate cancer. With a positivepredictive value of only $24.1 \%$ many cancers are likely to be detected and treated that do not - and would likely never - cause significant risk of patient mortality $[15,16]$. The American Prostate, Lung, Colorectal, Ovarian screening trial (PLCO) examined 79,693 men (55 - 74 years) who received annual PSA screening and found, at 13-year follow-up, very low prostate cancer mortality rates in both PSA-screened and non-screened groups; results were not statistically significant from each other [17]. Conversely, the European Randomized Study of Screening for Prostate Cancer (ERSPC) trial similarly evaluated 182,160 men (50-74 years) and found that at 11-year follow-up the rate of prostate cancer death was significantly reduced in the PSA-screened group [18, 19]. Conflicting trial results have left screening frameworks largely up to consensus decision from organizations, such as the National Comprehensive Cancer Network (NCCN), or the American Cancer Society (ACS) $[16,20]$. These studies demonstrate the controversial nature and ongoing debate around PSA screening; however, despite the controversy surrounding its use as a screening tool, PSA remains a valuable tool in the diagnosis, staging, and treatment selection of localized prostate cancer. 


\section{The prostate}

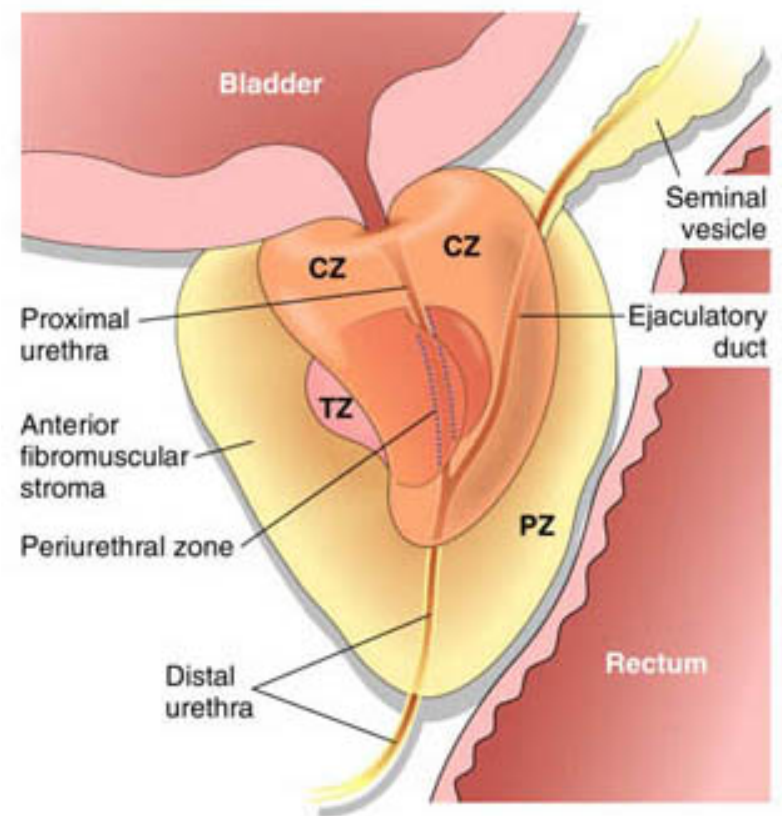

Figure 1.2: Zones of the prostate where cancer typically develops.

Abbreviations: $\mathrm{CZ}=$ Central Zone, $\mathrm{TZ}=$ Transition Zone, $\mathrm{PZ}=$ Peripheral Zone. Ref: http://alancam.com/study/path/view.php?url=Prostate\%20-\%20Pathoma.html

\subsubsection{Diagnosis \& Staging}

An elevated PSA screening result is not itself indicative of a diagnosis of prostate cancer. Several additional evaluations need to be undertaken by physicians to identify prostate cancer and determine its severity. Diagnosis and staging of prostate cancer is commonly performed by physicians using clinical nomograms or risk groups such as the NCCN prostate cancer risk stratification system. The American Joint Commission on Cancer (AJCC) TNM staging system also groups cancers by clinical factors such as the extent of the primary tumor $(\mathrm{T})$, presence of disease in the lymph nodes $(\mathrm{N})$, or presence of distant metastases (M); many of these are determined by physical evaluation or diagnostic tests. Ultimately, the staging of the disease into risk groups is used to a) inform appropriate therapies, and b) offer a prediction about the prognosis following therapy [21]. Characterization of prostate cancer is commonly done using several metrics including the clinical stage (TNM classification), and a measure of cellular pathology called the Gleason Score (GS), in addition to the serum PSA. 
The TNM stage, or clinical disease stage, is generally the first step in diagnosing and staging prostate cancer; a physician will typically determine this feature by palpation through a digital rectal exam (DRE). Following clinical staging the patient may undergo an imaging workup (which may include bone scans, Computed Tomography (CT), or Magnetic Resonance Imaging (MRI) scans) to determine the extent of the primary disease or the presence or absence of metastatic disease [21]. Trans-Rectal Ultrasound (TRUS)guided biopsies are then obtained to determine the GS of the disease, which indicates the degree of atypical cells present upon histopathological analysis. Biopsy samples are assigned scores from 3 to 5 , with 5 representing very poorly differentiated cancerous cells that significantly differ from the normal cellular structures. A pathologist will typically examine the two most common types of cellular patterns present in the biopsy specimen. Scores are assigned to each of the biopsy specimens; the total GS is reported as the sum of these two values, higher GS values correlate with more aggressive tumors [22]. Incorporated holistically the information obtained from these separate sources of clinical information is used to not only diagnose prostate cancer but inform the disease stage; Table 1.1 shows the NCCN guidelines for risk stratification of prostate cancer. The subsequent sections will discuss treatment options for localized prostate cancer; localized disease, as defined by the NCCN guidelines, refers to prostate cancer confined to the prostate gland and is typically restricted to Gleason scores of $\leq 7$.

\subsection{Management of Localized Prostate Cancer}

\subsubsection{Active Surveillance}

Due in part to potential over-screening among the general population, and therefore increased detection of early-stage prostate cancer, it has been estimated that as many as $23 \%-42 \%$ of PSA-detected cancers are over treated despite no significant evidence for long-term survival benefits of active therapy [23, 24]. Active Surveillance (AS) involves the periodic follow-up of patients at risk for development of more aggressive disease and may include repeat biopsies, but does not otherwise include active treatment; it differs 
Table 1.1: NCCN risk stratification groups for prostate cancer [21].

Abbreviations: $\mathrm{T}=$ Tumor volume, $\mathrm{N}=$ Node status, $\mathrm{M}=$ Metastatic, PSA = ProstateSpecific Antigen, $\rho \mathrm{PSA}=\mathrm{PSA}$ density, GS $=$ Gleason Score, $G S_{1}=$ primary GS pattern, $\mathrm{BxC}=$ Biopsy Cores, $\mathrm{Ca}=$ Cancer

\begin{tabular}{|c|c|c|c|c|c|c|c|}
\hline $\begin{array}{l}\text { Disease } \\
\text { Extent }\end{array}$ & $\begin{array}{l}\text { Risk } \\
\text { Group- } \\
\text { ing }\end{array}$ & $\mathrm{T}$ & $\mathbf{N}$ & $\mathrm{M}$ & $\begin{array}{l}\text { PSA } \\
(\mathrm{ng} / \mathrm{mL})\end{array}$ & $\begin{array}{l}\text { Gleason } \\
\text { Score }\end{array}$ & Notes \\
\hline Localized & $\begin{array}{l}\text { Very-Low } \\
\text { Risk }\end{array}$ & T1c & N0 & M0 & $\begin{array}{l}<10, \\
\rho \mathrm{PSA} \\
<0.15 \\
\mathrm{ng} / \mathrm{mL} / \mathrm{g}\end{array}$ & $\leq 6$ & $\begin{array}{l}<3+\mathrm{BxC},< \\
50 \% \mathrm{Ca} / \mathrm{BxC}\end{array}$ \\
\hline Localized & Low Risk & $\begin{array}{l}\text { T1a- } \\
\text { c, } \\
\text { T2a }\end{array}$ & N0 & M0 & $<10$ & $\leq 6$ & None \\
\hline Localized & $\begin{array}{l}\text { Intermediate } \\
\text { Risk }\end{array}$ & $\begin{array}{l}\text { T2b- } \\
\mathrm{c}\end{array}$ & N0 & M0 & $10-20$ & $\leq 7$ & None \\
\hline Localized & High Risk & T3a & N0 & M0 & $>20$ & $\geq 8$ & None \\
\hline $\begin{array}{l}\text { Locally } \\
\text { Advanced }\end{array}$ & $\begin{array}{ll}\text { Very } & \text { High } \\
\text { Risk } & \\
\end{array}$ & $\begin{array}{l}\text { T3b, } \\
\text { T4 }\end{array}$ & N0 & M0 & Any PSA & $\geq 8$ & $\begin{array}{l}G S_{1}=5,>4 \\
\mathrm{BxC} G S_{1}>8\end{array}$ \\
\hline Metastatic & $\begin{array}{l}\text { Metastatic } \\
\text { Disease }\end{array}$ & $\begin{array}{l}\text { Any } \\
T\end{array}$ & N1 & M0 & Any PSA & Any GS & None \\
\hline Metastatic & $\begin{array}{l}\text { Metastatic } \\
\text { Disease }\end{array}$ & $\begin{array}{l}\text { Any } \\
\mathrm{T}\end{array}$ & N1 & M1 & Any PSA & Any GS & None \\
\hline
\end{tabular}

from Watchful Waiting (WW) which includes no periodic biopsies as part of disease management [25]. Patients suitable for AS generally fall into the very-low-risk category. Although a viable method to avoid the over-treatment of low-risk prostate cancer there exist challenges in implementing this approach as a standard-of-care. A recent study by Klotz et al (2010) showed that for patients under AS the overall survival rate was $78.6 \%$ at follow-ups ranging from 1 to 13 years; over 30\% eventually required treatment which was ultimately less successful than if therapy was initiated from the onset of disease detection (50\% biochemical failure rates at 3 years) [26]. The findings of Klotz et al. indicate that although AS can provide an effective means to reduce overtreatment of prostate cancer there is a risk of missing more aggressive disease.

Studies also demonstrate that there may be considerable anxiety for patients harboring active cancer regardless of the risk with many patients opting-in for active treatment $[27,28]$. Coupled with a largely poor consensus on what differentiates cancers at risk of 
causing mortality from more indolent cancers [29, 30], and a risk of under-staging disease [29], there may be considerable risk of not actively treating younger men that may benefit from longer, disease-free lifespans.

\subsubsection{Active Treatment}

Definitive treatment for localized prostate cancer is primarily based on the risk group the patient occupies (see Table 1.1). Additional considerations for treatment selection include the patient's age, urinary function, and presence or absence of other comorbidities [25]. A large systemic review by Grimm et al. (2012) compared the effectiveness of various treatment options in terms of long-term disease control (see Fig 1.3) for localized prostate cancer including radical prostatectomy (or surgery), permanent and temporary brachytherapy, and External Beam Radiation Therapy (EBRT) [31]. The following sections outline the most commonly used techniques for the treatment of localized prostate cancer.

\section{Surgery}

Radical Prostatectomy (RP), the surgical removal of the prostate gland, has been the most historically utilized approach to treating organ-confined prostate cancer. Due to downward stage migration from increased PSA screening practices there has been increased viability in the use of surgical treatment options. Surgery is generally recommended for patients younger than 70 years of age with a life expectancy of greater than 10 years, and with no significant confounding urinary comorbidities [25]. Because RP is a surgical procedure the skill with which the technique is performed is highly important in obtaining good outcomes $[32,33]$. Complications can occur including significant urinary incontinence, erectile dysfunction, and rectal tissue injury related to operative damage to the respective tissues; however, the severity may be highly dependent on the skill of the surgeon [33 - 35]. The skill dependent nature of outcomes following RP was demonstrated in a retrospective review by Crook et al. (2015) which showed disease-free rates at followups of up to 15 years as low as $60 \%$ to $68 \%$, at different centers, for intermediate-risk 


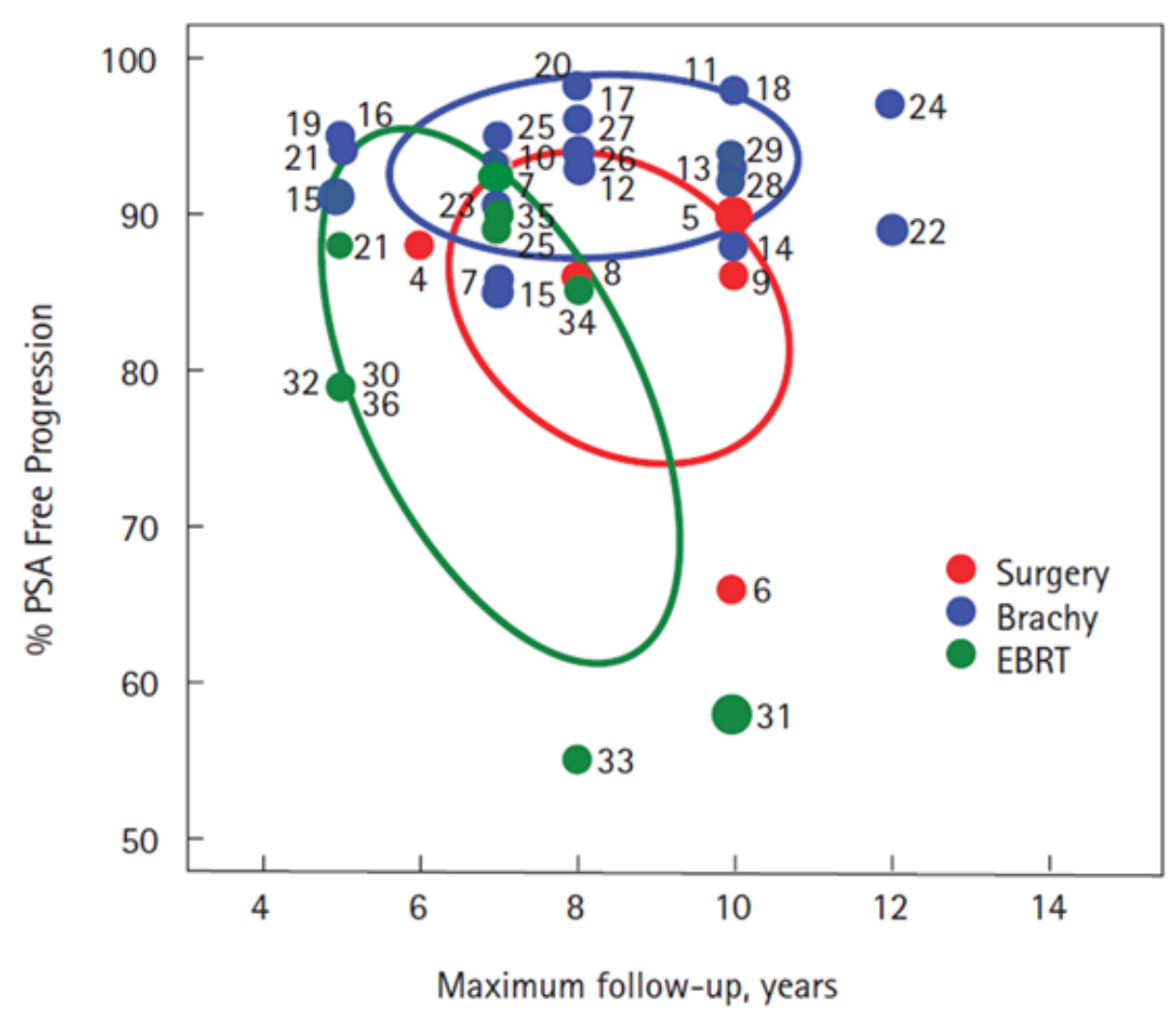

Figure 1.3: Results from a systemic review showing biochemical control rates for low-risk prostate cancer. Ellipses represent 1 standard deviation about the weighted mean of the data sets. Numbers over data points represent the study reference number [31].

Abbreviations: EBRT $=$ External Beam Radiation Therapy, Brachy $=$ Brachytherapy, PSA $=$ Prostate-Specific Antigen

patients [36].

\section{External Beam Radiation Therapy}

External Beam Radiation Therapy (EBRT) is another well-established treatment for localized prostate cancer. It can additionally be combined with other treatment modalities to offer improved disease control in higher risk patients. Several studies on the radiobiological nature of prostate cancer cells indicate that improvement in the therapeutic ratio (the balance between cancer control and normal tissue complications) is possible by delivering larger localized radiation doses in shorter time frames [37, 38]. EBRT is relatively less expertise-dependent compared to RP in that a) clinician skill is not correlated to treatment outcome, and b) relatively few contraindications exist to its use. Five-year follow-ups showing biochemical control of up to $90 \%$ in low-risk patients are common 
with relatively low rates of urinary toxicities $[31,39]$. Limitations of this approach when used alone show higher rates of rectal toxicities and increased local failures on long-term follow-up [39]. In comparison to brachytherapy and RP, which are single day procedures, EBRT is commonly delivered daily over $4-5$ weeks which may have a greater impact on a patient's quality of life.

\section{Brachytherapy}

Brachytherapy (BT) - a form of localized, internal radiation therapy where small radioactive sources are implanted directly within or near the cancer - is one of the most effective methods for the treatment of early-stage prostate cancer. Brachytherapy can either be delivered as permanent implants, Low-Dose-Rate (LDR) brachytherapy, or as temporary implants, High-Dose-Rate brachytherapy (HDR) brachytherapy. In LDR brachytherapy 80 - 100 small seeds commonly containing Iodine-125 (I-125) are implanted directly into the prostate gland in a pre-defined geometric pattern. The sources deliver the prescribed dose of radiation to the tumor as they decay. More recently HDR brachytherapy has emerged which offers a more versatile method of boosting the localized radiation dose in a safe, effective manner. In HDR brachytherapy a single Iridium-192 (Ir-192) source is remotely "stepped" through a series of 12-16 catheters temporarily implanted within the prostate gland, the dwell times and positions of this source within the catheters determines the radiation distribution within the prostate [40,41]. Although HDR brachytherapy offers promise as a modality LDR remains the standard of care, with a significant body of evidence that has yet to mature in the HDR setting.

LDR brachytherapy can either be delivered alone (monotherapy), or in combination with EBRT for high-risk disease [42]. Biochemical disease control, as measured by serum PSA at 5-7 year follow-ups, are typically over 95\% in low-risk disease, and greater than 90\% in studies evaluating intermediate-risk patients [43-45]. LDR brachytherapy also has demonstrated relatively low morbidity rates as measured by acute and long-term urinary and rectal toxicities in a number of studies, with the best sexual function scores among all the available treatment options [35, 42, 46]. An excellent prospective study by 
Giberti C et al. (2009) compared brachytherapy to RP for low-risk patients and found nearly identical biochemical survival (91\% and 91.7\%, respectively), but less frequent erectile dysfunction in the BT group; although, at 5-year follow-up the rate of longterm side-effects was nearly identical [47]. Quality-of-life indices also show significant benefits to LDR brachytherapy over RP for localized disease [48]. Because of these benefits LDR brachytherapy is routinely recommended for the treatment of low-risk disease by the American Brachytherapy Society (ABS) and Groupe Européan de Curiethérapie European Society for Radiotherapy and Oncology (GEC-ESTRO) organizations, as well as the ACS and American Urological Association (AUA).

Due to the ability to implant sources directly within the cancerous lesion the movement of the target organ becomes inconsequential to treatment delivery (unlike EBRT, where motion is a significant concern during treatment), thereby allowing significant, and safe, radiation dose escalation to the cancer. The goal of LDR brachytherapy is therefore to maximize the radiation dose received by the cancer while limiting radiation dose to nearby healthy tissues such as the rectum and urethra. As this thesis will demonstrate this process is more nuanced and complex than its initial simplicity implies.

\subsection{Current Issues in Brachytherapy}

\subsubsection{The Low-Dose-Rate Workflow}

Fig 1.4 shows the modern TRUS-guided LDR brachytherapy implantation procedure as developed by Stock and Stone et al in 1995 [49]. Patients are typically sedated using general anesthesia and placed in dorsal lithotomy position within an operating suite. A volumetric TRUS image is then acquired. In the preoperative planning workflow the patient is then discharged and treatment planning proceeds in their absence; in the intraoperative planning workflow the brachytherapy plan is generated and implanted while the patient is in the operating room. During treatment planning a radiation oncologist with expertise in brachytherapy (also known as a brachytherapist(BT)), will use the TRUS volume information to identify and contour the target and normal tissues, or Organs-at- 


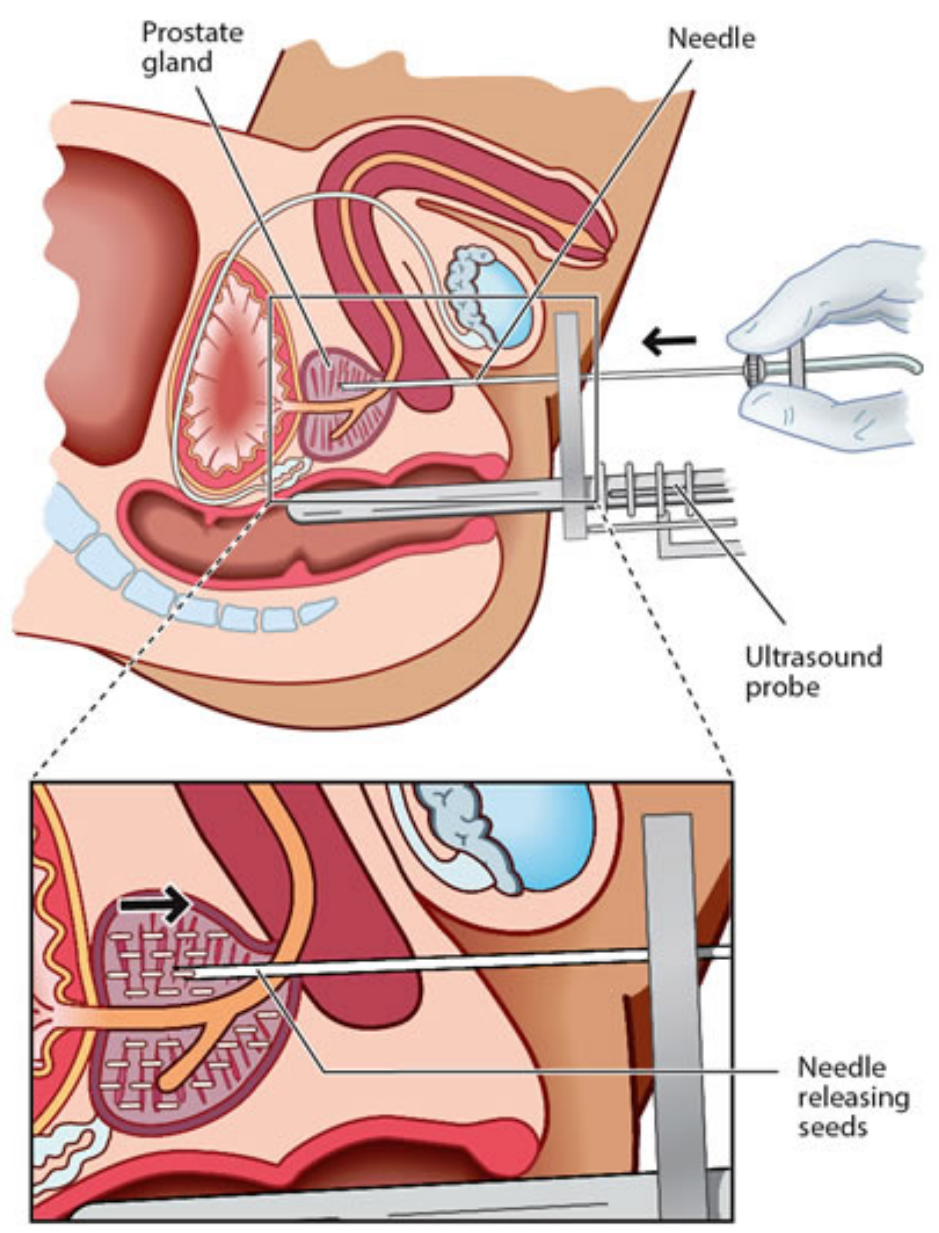

Image reproduced with permission of Prostate Cancer Foundation of Australia.

Figure 1.4: The modern TRUS-guided prostate LDR brachytherapy procedure. Radioactive sources (contained within small metallic seeds) are deposited into the prostate using hollow needles. Ref:http://www.prostate.org.au/awareness/for-recently-diagnosed-menand-their-families/localised-prostate-cancer/treatment/treatment-options/

Risk (OAR). Once the contoured organ structures are identified the task falls to an expert treatment planner (either a BT or an expert Radiation Therapist / Dosimetrist (RT)) to manually place the radioactive sources in an optimal 3D configuration to deliver as much radiation to the target as possible while minimizing dose to normal tissues. A dose of 110 Gy - 145 Gy is typically prescribed to the prostate (for boost and monotherapy treatments, respectively) [40]. Following planning the radiation oncologist then implants the planned configuration of I-125 sources under TRUS-guidance using pre-loaded or custom loaded needles. The implantation process causes swelling and edema of the prostate gland and alters its shape. Post-implant dosimetric analysis is typically performed 14 to 30 days after the implant procedure to allow sufficient time for swelling to subside; post-implant 
dosimetry done immediately after implantation can underestimate the dose by as much as 10\% compared to day 30 [49]. During post-operative dosimetry the patient undergoes a CT scan and the sources are identified, the plan dosimetry is then recomputed from the actual positions of the sources.

Iodine-125, the most common isotope used in prostate brachytherapy, decays by electron capture to an excited state of Tellurium-125. The emission of low energy (average 35.5 $\mathrm{keV})$ gamma (7\%) and characteristic x-ray (93\%) photons follows decay with a half-life of 59.4 days; these short-range photons transfer energy to cancerous tissue causing strand breaks in the DNA of cells [51]. During treatment planning specialized software takes the position of these sources, along with the contours provided by the radiation oncologist, and computes the dose to the target (prostate) and OARs. Dose computation is based on the American Association of Physicists in Medicine Task Group No. 43 formalism and is standard practice for brachytherapy planning software; this formalism will be covered in greater detail in a subsequent section $[40,52]$. With the dose computed and a set of physician-defined contours the treatment plan is evaluated using a Dose-Volume Histogram (DVH). This measure graphically represents the volume of the prostate contour receiving a given dose of radiation. For example, D90 represents the amount of dose received by $90 \%$ of the prostate; conversely, V100 may be used and refers to the prostate volume receiving $100 \%$ of the prescribed dose. These metrics, in particular the prostate D90, have been correlated to outcomes in biochemical control for both the preoperative treatment plan and post-operative dosimetry [40, 53, 54].

\subsubsection{Limitations of Treatment Planning Methodologies}

Post-implant dosimetric and clinical outcome studies demonstrate that the longterm disease control and side-effects from LDR brachytherapy are highly dependent on the expertise of the brachytherapy team and the quality of the implant $[55,57]$. LDR brachytherapy plans are more susceptible to seed misplacement during the implantation procedure and result in less frequently achieving adequate target coverage and normal tissue sparing [57, 58]. A study conducted by Morton et al. (2013) showed a larger 
variance in dosimetric quality for prostate V100 and V200 metrics for LDR compared to HDR brachytherapy [57]. A dosimetric learning curve for reducing long-term rectal [59], and urethral [60, 61], toxicities has been also demonstrated in several institutional studies. Multivariate analysis in a study by Liu HW et al. (2010) showed that the experience of the team may be the most important predictive factor in obtaining a quality dosimetric outcome, followed closely by the shape and size of the prostate gland itself [62]. Overall, the differences in LDR planning expertise across centers may be a cause of the observed lack of uniformity in both dosimetric results and clinical outcomes.

A significant number of cases with poor post-operative dosimetery are caused by systematic source placement errors during the implantation process [63, 64]. Poorly designed treatment plans will systemically propagate into poorly performed implants, these cases may require some form of salvage therapy or re-implantation to improve target coverage [63]. Due to this inherent connection between treatment planning and clinical outcomes it may be possible to improve the overall quality of the implant, and reduce the risk of improper implantation, by creating high quality treatment plans to begin with.

Sylvester et al. (2011) showed that a major contribution to the success of the implanted plan distribution is the three-dimensional source pattern implanted by the radiation oncologist [65]. Several features differentiate the expert plan from the novice plan: sources and needles (used to implant sources) are evenly distributed to avoid dose inhomogeneities, the number of needles is kept to a minimum to reduce implant edema and ease visualization on TRUS imaging [60, 66], and, sources are strategically placed to limit plan distortion as a result of post-implant edema [66]. Expert brachytherapy planners will take into account this experiential knowledge of the implant process, in addition to the skill of the BT in implanting the case, to minimize the likelihood of producing a poor implant and deviating from the planned source pattern. The large number of potential source positions, and the multitude of both clinical and dosimetric parameters to consider, make planning resource intensive and time-consuming; seasoned experts may take anywhere from 20 minutes to several hours to construct a high-quality treatment plan. In order to improve plan quality, improve the uniformity of treatment planning and clinical outcomes 
in LDR brachytherapy, and decrease the risk of poorly performed implants new methods of performing this crucial workflow step are needed.

\subsection{Potential Solutions to the Planning Problem}

\subsubsection{Overview of Strategy to Be Implemented}

The optimal selection of radioactive sources relative to the prostate and normal tissues is presented as a difficult optimization problem with a multitude of clinical and dosimetric variables to consider. As computational power increases there exists opportunities to apply computer algorithms to solve these complex, multifactorial problems.

Anatomy based inverse planning methods to optimize plan dosimetry have been successfully used in EBRT to rapidly produce high quality treatment plans [67]. Anatomy based inverse optimization refers to the use of radiation oncologist-defined organ contours and dosimetric objectives as inputs; the optimal radiation distribution from these known inputs is then calculated using a number of different methodologies [68, 69]. Dinkla AM et al (2015) showed that inverse optimization may offer comparable dosimetric results to expert-generated brachytherapy treatment plans but with the added benefit of significantly decreased planning times [70]. Several computerized algorithms have been developed including the Inverse Planned Simulated Annealing (IPSA) algorithm developed by Pouliot (1996) and further improved by Lessard et al [71-73], the Hybrid Inverse Planning and Optimization (HIPO) algorithm which combines stochastic and deterministic processes [74], mixed-integer methods [75], and several variations of simple, multi-objective genetic and evolutionary algorithms [76, 77]. Unfortunately many of these algorithms are experimental and have seen limited clinical use on their own, particularly in LDR brachytherapy.

In the following sections one of these algorithms, the fast IPSA implementation, will be examined including its benefits in dosimetric optimization for brachytherapy but also its deficiencies when used without supplemental clinical information. 


\subsubsection{Stochastic Optimization}

The most successful of the stochastic algorithms used in LDR brachytherapy is the IPSA algorithm. Its application in LDR brachytherapy has many advantages including its ability to escape the many local optima that exist among LDR treatment plans to increase the likelihood of finding an overall optimal solution. The core simulated annealing algorithm, as described by Kirkpatrick (1983), can be understood as analogous to a slowly cooling liquid (annealing); as the energy state of the liquid decreases, due to a reduction in the ambient temperature, it will eventually solidify into a lattice formation representing its most stable (lowest energy) form. If cooling is too rapid the liquid may not have sufficient time to form an ordered lattice; an amorphous solid is the result which may not necessarily correspond to the lowest and most stable energy state [78]. In this implementation the energy state is defined by an objective penalty function describing the source pattern and dosimetry of the treatment plan, as well as how close the plan is to some pre-defined dose objective. As with any optimization algorithm the goal is to minimize this function through a series of iterative, randomized steps and achieve some optimal plan dosimetry.

Pouliot et al (1996) defined the components of the IPSA algorithm relevant to LDR brachytherapy optimization which will be briefly covered in this section [71]. These components include 1) a detailed modeling of the treatment space and input data, 2) a method to compute the dose to the BT-defined targets and normal tissues, and 3) an objective penalty function, based on the dosimetric state of each organ, which must be minimized. Further details of this implementation will be covered in Chapter 2.

\section{Data Input}

The IPSA algorithm first requires the target and normal tissue contours, an initial placement of needles (generally also defined by the BT), and an initial source pattern. Typically $\boldsymbol{A}=\left[a_{1} \ldots a_{i}\right] \in \boldsymbol{R}$ represents a linearly-indexed vector for each contour point being evaluated, where $a_{1} \ldots a_{i}$ defines the linear indices of the dose points of the surface of the prostate (as an example). Similarly, $\boldsymbol{B}=\left[b_{1} \ldots b_{j}\right] \in \boldsymbol{R}$ represents the initial source 
pattern as a vector, where $b_{1} \ldots b_{j}$ define the linear voxel indices of each source centroid.

\section{Dose Computation}

The dose to each voxel of the prostate contour is computed from the TG-43U1 pointsource formalism [52]. This dose formalism has become standard in a number of commercially available dose computation engines due to its relative speed and ease of implementation. The one-dimensional point-source formalism is used to simplify computation by removing the need to determine source orientation; this can be described by the following:

$$
\dot{D}(r)=S_{k \delta} \Lambda\left(\frac{r_{0}}{r}\right)^{2} g_{p}(r) \phi_{a}(r)
$$

Where $\dot{D}(r)$ represents the dose rate to any point at a distance $\mathrm{r}$ from the centroid of the source (see Fig 1.5). The air-kerma strength is defined by $S_{k \delta}$ and is the air kerma rate in vacuo due to photon energies greater than an energy cut-off $\delta$ (typically $5 \mathrm{keV}$ ), the dose-rate constant in water is represented by $\Lambda$ and is the ratio of the dose rate at $1 \mathrm{~cm}$ on the transverse axis of the source $\left(r_{0}\right)$ to the air-kerma strength. An inverse-square law parameter is used to represent the isotropic dose fall-off from the point-source. Finally, the radial dose function $g_{p}(r)$, and the anisotropy function $\phi_{a}(r)$ represent interpolated values from experimental data that account for photon scatter and dose variations, respectively.

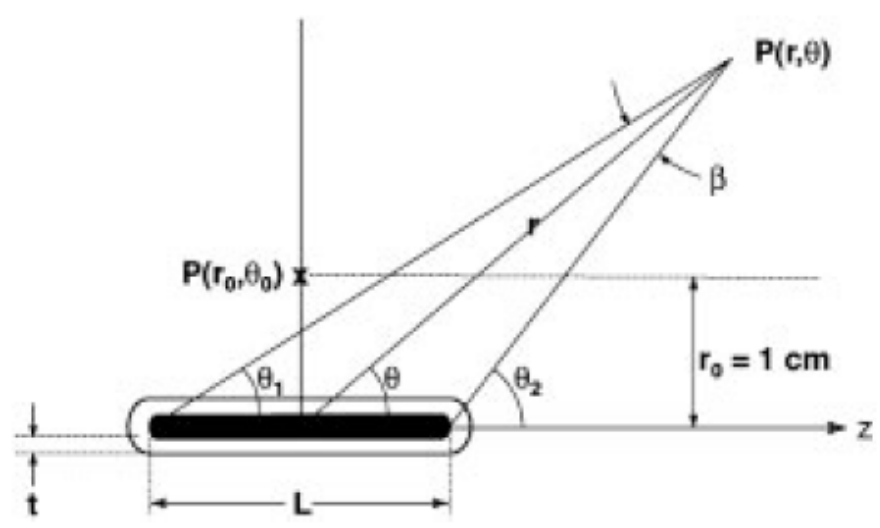

Figure 1.5: Generic line-source brachytherapy seed with computation parameters. The angular terms are not present for point-source approximations [52]. 
Given some initial source pattern $\boldsymbol{B}$, the dose to each voxel on any contour surface can be defined by $\boldsymbol{D}=\left[d_{1} \ldots d_{k}\right] \in R^{2}$ where $d_{1} \ldots d_{k}$ represents the linear indices of dose voxels computed on each prostate surface point from the $1^{\text {st }}$ to the $k^{\text {th }}$ voxel index. An example of the computed dose to the surface of the prostate can be found in Fig 1.6.
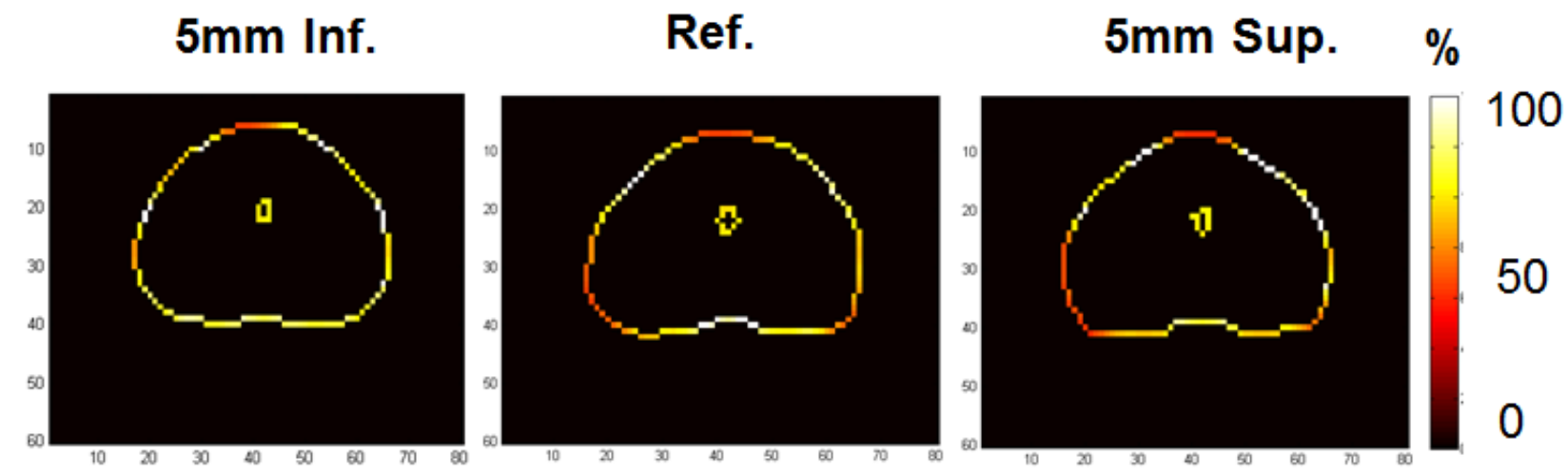

Figure 1.6: Example of dose computation from a given source pattern to the prostate and urethral contour surfaces. Dose is represented as a \% of the prescribed dose. Dimensions are in $\mathrm{mm}$.

Abbreviations: Sup $=$ Superior, Inf $=$ Inferior, Ref $=$ Prostate Midplane

\section{Objective Penalty Function}

With the known dose to the prostate contour an objective penalty function can be obtained that penalizes source patterns with dosimetric criteria not matching the physician's standards. Given the dose to the prostate contour represented by $\boldsymbol{D}$, a penalty score was assigned such that $\boldsymbol{O}(\boldsymbol{B})=\left[o_{1}, \ldots, o_{k}\right] \in \boldsymbol{R}$ where $o_{1}, \ldots, o_{k}$ represents the objective penalties assigned to each dose voxel (for all $k$ voxels), for a given source pattern. A physician-defined penalty may state that the prostate surface dose cannot be below 145Gy and set this as a relatively hard constraint that must be met. Similar constraints are applied to normal tissue contours. A more detailed explanation of the objective penalty function as it relates to novel algorithmic implementations is found in Chapter 2.

Once an objective penalty has been defined for each individual dose voxel of the prostate the organ-specific objective function can be defined from the following:

$$
O(B)_{\text {prostate }}=\frac{1}{N} \sum_{k=1}^{N} O(B)_{k}
$$


where $1 / \mathrm{N}$ represents the normalization of the summed voxel penalties to the total number of voxels computed. This type of equation is known as a multi-objective function minimization since each organ structure has a summed objective penalty function [79]. The most recent renditions of IPSA further simplify this minimization problem by creating a single-objective penalty function. The single-objective penalty function in this case represents the cumulative sum of objective penalty functions from 4 different objectives: the prostate surface and contour dose, the PTV dose, and the urethral doses. In summary, the iteration through different source patterns tries to balance these competing interests to find an optimal source pattern.

\subsubsection{Deficiencies of Naive Stochastic Optimization}

Despite the relative success of the fast IPSA implementation there exist several limitations to the naive implementation, including limitations common to all stochastic methods. Due to the limitations of these approaches IPSA (and other stochastic methods) have seen limited use; manual, expertise-driven planning still remains by far the most common approach in LDR brachytherapy.

1. Plans may not be physically implantable in patients. The stochastic (or random) nature of the source patterns may produce plans which are dosimetrically optimal but not achievable in clinical practice by even experienced radiation oncologists. Fig 1.7 outlines a dosimetrically optimal IPSA plan that demonstrates difficult implant features. Sources clustered in close proximity or to one side of the prostate may cause excessive hot spots to nearby normal tissues and may be exceedingly difficult to visualize under intraoperative TRUS-guidance due to a number of artifacts [80]. Plans with these features must generally be heavily modified to be implanted accurately by the physician. Additionally, to obtain plans similar to Fig 1.7 significant priming of the algorithm was required (for example using a prior expert physician-designed template).

2. Plan modification is difficult if not impossible. Because stochastic algorithms generate randomized source patterns re-optimizing the algorithm will degrade the 


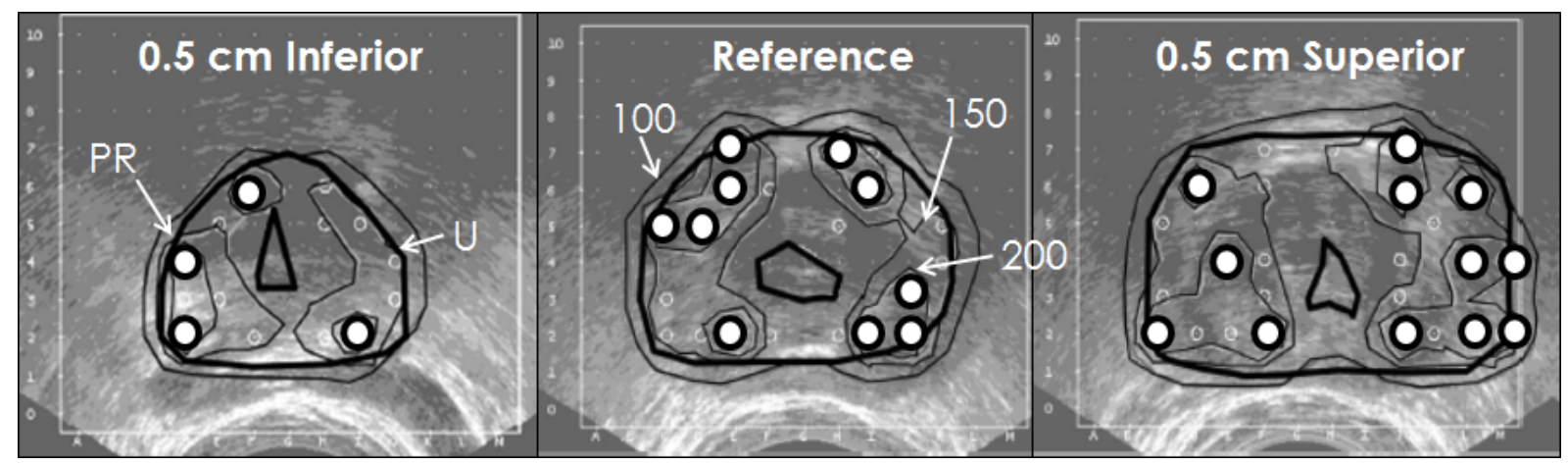

Figure 1.7: Treatment plan axial isodose distribution demonstrating optimized dosimetry but features that may cause problems during implantation. Isodoses are labelled as a percentage of the prescription dose [72]. Contours: $\mathrm{PR}=$ prostate, $\mathrm{U}=$ urethra. IPSA = Inverse Planned Simulated Annealing

initial source pattern altogether; there is no method to introduce minor modifications if required. Minor modifications to source patterns are difficult as the plans degrade in quality with deviations from the optimized source positions (i.e. they are optimized only to their current state). Adjustments by a BT or RT are therefore difficult and relegated to only altering hyperparameters of the algorithm. Hyperparameters refers to parameters that may be altered manually including the optimization time, prescribed dose etc, but typically are not tuned through iterative optimization.

3. Plan uniformity is poor. It follows from the limitations described in 1 and 2 that there exists poor uniformity in design of treatment plans, even for prostate glands with similar shapes and sizes to previously planned cases. As such, many clinical hours may be spent re-planning cases that were previously successful. There may exist significant differences in plan quality between expert planners and even between different cases of a single expert planner.

\subsubsection{Incorporation of Machine Learning}

A promising solution to the limitations imposed by stochastic optimization processes has emerged in recent years in the form of Machine Learning (ML) algorithms. Machine learning is a form of artificial intelligence that uses past information (in the form of historical training data) about a given problem to infer new solutions without being directly 
programmed for such an endpoint [81]. ML algorithms have been able to outperform conventional computation methods in complex language classification tasks, financial trading algorithms, video game artificial intelligence, and even automated diagnosis in radiology applications [82-84]. The ability to include prior information allows incorporation of human-like knowledge of patterns and solutions to provide more uniform and real-world solutions; the result is an algorithmic process that can typically mimic human modes of thinking, potentially even an expert BT or RT planner [85].

There is surprising variability in this broad group of algorithms where form generally follows function, i.e. the algorithmic workflow is tuned to the task it is trying to perform [86]. Algorithms requiring accuracy may require considerable processing to extract relevant information from a dataset, while those requiring speed may opt for simpler assumptions and faster optimization algorithms. The majority of machine learning algorithms contain the following components [86]:

1. Database preprocessing and database creation. Previous successful solutions to a problem are aggregated into a common training database.

2. Dimensionality reduction and similarity matching. Database entries are generally complex so a method of simplifying the data is needed. Principle Component Analysis (PCA), Mutual Information (MI), and primitive feature extraction are common methods of reducing complexity of data [87]. Similarity matching is used to find mutual information between a current problem and a previous solution from the database.

3. Iterative optimization. The most similar solution(s) is adapted to the current problem using a set of environmental constraints combined with iterative stochastic optimization.

4. Model revision \& training. Solutions are evaluated and successful attempts at the problem are retained for future use; the learning model is usually updated to reflect the methodology taken to reach the solution.

Since the main limitation of current LDR BT algorithms is their inability to replicate 
human-expert qualities machine learning may be able to replicate features in treatment plan design that resemble those of an expert treatment planner. When combined with the rapid adaptation process offered by stochastic optimization algorithms such as IPSA it may be possible to rapidly find optimal planning solutions that satisfy a multitude of competing dosimetric and clinical criteria. Chapter 2 will examine this hybrid approach to planning using a novel, brachytherapy-specific, machine learning algorithm.

\subsection{Hypothesis}

The goal of this thesis is to develop and evaluate the clinical efficacy of a novel machine learning approach to treatment planning for LDR prostate brachytherapy. This approach has the potential to vastly improve the resource and time requirements of expertise-driven planning, while simultaneously improving on the limitations of current computerized inverse planning methods. The incorporation of this approach into the clinical workflow is expected to produce results comparable to that of highly seasoned brachytherapy planners, in much shorter timeframes.

The main hypothesis of this work is that implementing this novel algorithm in the prostate LDR brachytherapy workflow can produce pre-implant treatment plans of comparable dosimetric and clinical quality to that of expert brachytherapy planners, in addition to significantly reduced planning times.

\subsection{Potential Impact}

It is expected that the results of this study will significantly impact the accessibility of high quality prostate LDR brachytherapy treatment planning. Firstly, this thesis has the potential to significantly reduce the usage of costly and clinical resources, such as the planning time required by brachytherapy expert planners; with the predicted increases in prostate cancer this may allow centers to reliably keep pace with growing demand for their services. Secondly, the thesis has the potential to redefine how brachytherapy is administered across centers. Several clinical trials $[57,88]$ show that outcomes in LDR 
brachytherapy although excellent require improved uniformity and consistency of results, particularly with relation to side-effects. The incorporation of standardized planning methodologies based on expert consensus allows for improved uniformity in treatment planning. Finally, the collection of increased volumes of brachytherapy outcomes data may also allow future insights to develop on the treatment outcomes which are not currently being collected. The work presented here has been adopted by MaRS ${ }^{T M}$ Innovation, an organization that specializes in early stage seed investing for developing companies, with the intention of making the algorithm readily available to clinical end users.

\subsection{Thesis Overview}

Chapter 2 describes the evaluation of a novel incorporation of ML into the LDR brachytherapy framework as a means to achieve the goals set out in the hypothesis. This chapter also covers in more detail the components of the algorithm, as well as the results of a clinical study conducted at the Odette Cancer Center (Toronto, ON, Canada). Chapter 3 includes an expanded discussion of how these findings will affect the nature of brachytherapy treatment planning in the future. 


\section{Chapter 2}

\section{Evaluation of a Machine Learning}

\section{Algorithm for Prostate LDR Brachytherapy}

\section{$2.1 \quad$ Abstract}

Purpose: This work presents the first known incorporation of machine learning (ML) to automatically generate high-quality, prostate Low-Dose-Rate (LDR) brachytherapy treatment plans. The ML algorithm has the ability to mimic implant characteristics of expert brachytherapists. The planning efficiency, dosimetric and clinical quality of plans generated using an ML planning approach was evaluated in this study.

Methods \& Materials: Pre- and post-implant treatment plans were extracted from 100 high-quality LDR treatments and stored within a training database. The ML algorithm matches similar features from a new LDR case to those within the training database to rapidly obtain an initial seed distribution; plans were then further fine-tuned using a stochastic local search algorithm. Pre-implant treatment plans generated by the ML algorithm were compared to brachytherapist (BT) treatment plans in terms of planning time (Wilcoxon rank sum, $\alpha=0.05$ ) and dosimetry (one-way ANOVA, $\alpha=0.05$ ). Qualitative 
clinical implant quality was evaluated by expert LDR radiation oncologists using a Likert scale questionnaire.

Results: The average planning time for the ML algorithm was $0.84 \pm 0.57$ min compared to $17.88 \pm 8.76 \mathrm{~min}$ for the expert planner $(\mathrm{p}=0.020)$. Treatment plans were dosimetrically equivalent to the BT plans; the average prostate V150\% was $4 \%$ lower for ML plans $(\mathrm{p}=0.002)$; although, not clinically significant. Respondents ranked the ML generated plans as equivalent to expert BT treatment plans in terms of target coverage, normal tissue avoidance, implant confidence, and the need for plan modifications. Respondents had difficulty differentiating between plans generated by a human or the ML algorithm.

Conclusions: Prostate LDR treatment plans that have equivalent clinical and dosimetric quality to plans created by brachytherapists can be rapidly generated using ML. The adoption of ML in the brachytherapy workflow is expected to produce high quality LDR treatment plans uniformly, while dramatically reducing planning time and resources.

\subsection{Introduction}

Low-Dose-Rate (LDR) prostate brachytherapy, the permanent implantation of radioactive sources directly within the prostate, is one of the most effective methods of delivering conformal radiation for localized prostate cancer [31,89-92]. Several clinical trials have demonstrated excellent long-term biochemical disease control using LDR brachytherapy, either alone (as monotherapy), or as a boost following External Beam Radiation Therapy (EBRT) [93-96]. The overall goal of LDR brachytherapy is to maximize the dose to the prostate while sparing normal tissues through the implantation of radioactive sources in a pre-determined three-dimensional pattern. Studies evaluating post-implant outcomes in LDR brachytherapy demonstrate that the quality and long-term success of the procedure are highly dependent on the expertise of the brachytherapy team $[55,56,97]$.

Hughes et al (2005) and Sasaki et al. (2014) demonstrated that systematic source placement error was often the cause of poor post-implant dosimetry; implants with regions of insufficient dose identified during post-implant evaluation have been associated with 
local recurrences $[63,64]$. Re-implantation following a suboptimal implant may improve target dosimetry but frequently results in significant urinary morbidity in addition to the increased operative risk [98]. The pre-implant treatment plan is a critical component to ensuring accurate implants; poorly optimized plans may often result in suboptimal implants as errors are systematically propagated throughout the procedure. Expert radiation therapist (RT) planners will often instinctively account for the skill of the brachytherapist (BT) and the particularities of the implantation technique to maximize the likelihood of an accurate implant. Although this has led to excellent results, this expertise-driven approach to LDR planning requires large patient volumes, is resource intensive, and timeconsuming. Recent evidence suggests that there may also be poor uniformity of outcomes due to different levels of procedural expertise $[57,88]$.

One method of ensuring uniform, high quality treatment planning has been the introduction of computer optimization algorithms. A study by Dinkla AM et al (2015) showed that inverse optimization algorithms can also significantly decrease planning time compared to expert-driven methods [70]. Inverse Planned Simulated Annealing (IPSA) [71], the Hybrid Inverse Planning Optimization (HIPO) algorithm [74], mixed-integer methods [75], and variations of multi-objective genetic algorithms have been examined to improve brachytherapy planning [76]. Conventional stochastic algorithms, although capable of rapid planning, commonly fail to incorporate the implantation technique of the BT into the planning process; thereby, producing plans that are difficult to reproduce during implantation [99]. Relatively few of these algorithms have been successfully implemented in the clinical environment owing to these limitations; stochastic optimization alone may therefore be insufficient to produce optimal treatment plans without supplemental information.

A promising algorithmic technique applicable to brachytherapy planning is machine learning. Machine learning (ML) is a form of statistical computation that uses known properties of historical data to predict solutions; these techniques have seen considerable interest in recent years [81]. Applications range from performing complex language classification tasks [82], to financial trading [83], to computer-aided radiological diagnosis [84,85]. The 
main benefit of such active learning approaches is that they can typically mimic human modes of thinking in highly complex tasks [100].

The ML paradigm is suited to the complex task of finding viable source patterns in LDR brachytherapy that account for BT skill and implant technique. This could provide significant planning assistance to overextended brachytherapy departments, increase the uniformity in treatment plan quality, and reduce the likelihood of systemic implant errors. To date there has been no published study involving ML for brachytherapy (to the authors's knowledge); this study presents the dosimetric and clinical results for the first known application of ML for prostate brachytherapy planning.

\subsection{Materials \& Methods}

\subsubsection{Data Description}

Data used to inform the ML algorithm was extracted from the LDR brachytherapy patient database at the Sunnybrook Odette Cancer Centre (Toronto, ON, CA) as part of an ethics board-approved study. The center treats approximately 100 prostate LDR cases per year and has a well-established prostate brachytherapy program. A random sample of 150 LDR cases dating back to 2012 was obtained. Cases were filtered according to postimplant dosimetric quality metrics, primarily the prostate D90\% and V100\% [56,101], to obtain an initial ML training database of 100 cases. In addition to planning information and structure contours, primitive anatomic features were computed and stored within the database. An additional 30 randomly selected LDR cases were used to validate the ML algorithm output.

\subsubsection{System Architecture}

Fig 2.1 demonstrates the workflow of the ML algorithm for creating clinically viable treatment plans. The core components of the algorithm required to generate a treatment plan can be deconstructed into three steps: 1) data preprocessing and training database creation, 2) similarity matching, and 3) stochastic optimization. 


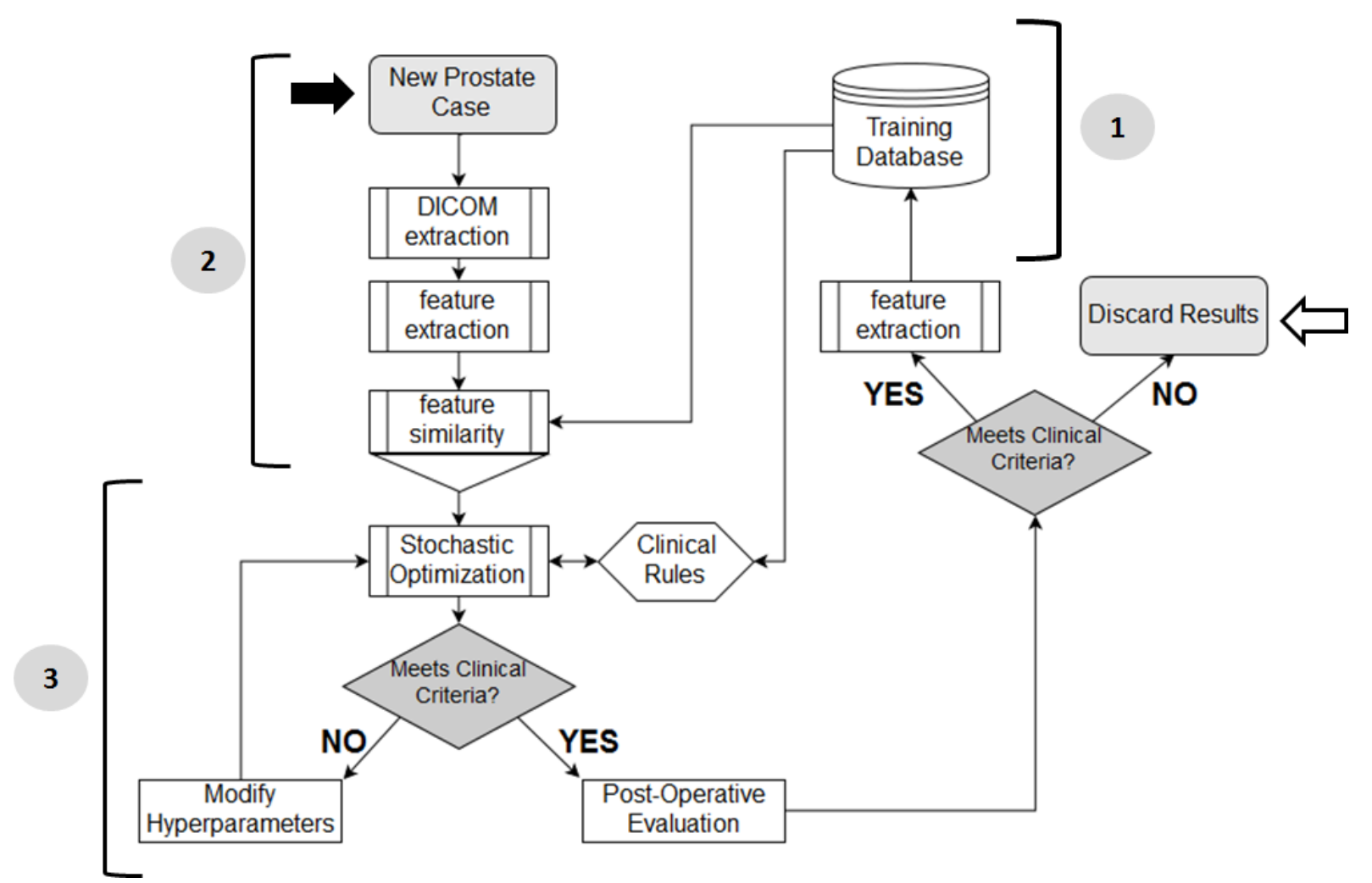

Figure 2.1: Machine learning workflow for LDR brachytherapy. 1) data preprocessing and training database, 2) similarity measure functions, 3) stochastic optimization and plan adaptation. The dark arrow represents the starting point; the white arrow represents a possible terminus. For a list of clinical rules used to constrain stochastic optimization refer to Table 2.1 .

\section{Data Preprocessing \& Training Database}

Digital Imaging and Communications in Medicine (DICOM) files from a new LDR case were first used to extract relevant information from the respective case. BT-defined contours (prostate, urethra, rectum and planning target volume), source patterns, and radioisotope properties were automatically extracted and stored as data within the training database. Primitive features - a lower-dimensional quantitative representation of the extracted contour structures - are a method to simplify the large amount of 3D contour data that exists $[87,102]$. Extracted features included the prostate volume, principal axes of the prostate, axial slice circumference, and prostate symmetry; many of these features are currently used in semi-quantitative approaches during prostate biopsies or treatment planning to characterize the prostate [103-105]. Features were z score normalized to remove the effect of varying units [106]. 


\section{Similarity Matching}

The similarity matching process was used to find the most similar features of cases from the training database which matched those of the current case. A Euclidean distance metric was used to inform a $k$-nearest neighbor $(k \mathrm{NN})$ plan retrieval method, this approach is useful when all data points are quantitative and the database is of limited size [107-109]. A vector representing the features for the query case was given by $\boldsymbol{X}=\left[x_{1} \ldots x_{i}\right] \in \boldsymbol{R}$ where $x_{1}, \ldots, x_{i}$ represents the first to the $i^{\text {th }}$ computed anatomic feature dimension. Similarly, a matrix representing the features for the database cases was given by $\boldsymbol{Y}=\left[y_{1,1} \ldots y_{i, j}\right] \in \boldsymbol{R}$ where $y_{1,1} \ldots y_{i, j}$ represents identical features computed for the first to the $i^{\text {th }}$ feature for each $j^{\text {th }}$ database plan. To find the similarity vector $\Pi=\left[\pi_{1} \ldots \pi_{j}\right] \in \boldsymbol{R}$

$$
\forall j \in[0, M], \quad \pi_{j}=\sqrt{\sum_{i=1}^{N}\left(x_{i}-y_{i, j}\right)^{2}}
$$

where $\pi_{1} \ldots \pi_{j}$ represents the sum of the distance values for the $i^{\text {th }}$ feature, for all $N$ features, between the query and database cases. Similarly, this was computed for all $M$ database cases to produce the similarity vector $\prod$. The $k \mathrm{NN}$ retrieval was then used to retrieve the top $k$ plans from the training database with the most similar anatomic features by finding the smallest elements of the similarity vector. The best matched plan(s) was populated onto the BT-defined contours prior to further optimization.

\section{Stochastic Optimization}

Due to the relative success of the IPSA algorithm by Pouliot et al (1996) in optimizing dosimetry this core methodology was used to adapt the most-similar plan to the anatomy of the new case. The IPSA algorithm uses minimization of a dosimetric objective function by rapidly evaluating multiple potential source patterns in relation to the defined contours. The smaller the objective function the increased likelihood that an optimal solution has been found [71].

The best matched plan(s) obtained from the training database was used to compute the 
dose to the contoured structure volumes using the TG43 point-source formalism [52]. The dose to each contoured structure was stored as a linearly-indexed vector $\boldsymbol{D}=\left[d_{1} \ldots d_{k}\right] \in \boldsymbol{R}^{3}$ for the first to the kth voxel within or on the surface of the structure volume. Each voxel element of the structure that was not within a predefined dose range was penalized according to

$$
O(A)= \begin{cases}\left|\left(d_{k}-D_{t}\right)\right| W & \text { if } d_{k}<D_{l} \text { or } d_{k}>D_{h} \\ 0 & \text { otherwise }\end{cases}
$$

where $d_{k}$ is the $k^{\text {th }}$ voxel dose for a given structure volume, $D_{t}$ represents either the higher $\left(D_{h}\right)$, and lower $\left(D_{l}\right)$ dose limits of a structure, $W$ is a weighting vector, and $O(A)$ represents the objective function score for a given source pattern, $A$. The hyperparameters $D_{h}$ and $D_{l}$ were obtained by BT consultation and remained constant. The values of the $\mathrm{W}$ vector were obtained using cross-validation on the initial training database [110]. Summing penalty scores for each voxel yielded the cumulative objective function for a given structure. In total, four objectives were considered: prostate and planning target volume (PTV) coverage, a uniformity constraint, and the urethral maximum dose. These represented soft-constraints for the algorithm to achieve through objective function minimization.

In addition to the soft-constraints provided by the dosimetric objective function several clinical rules were used as hard-constraints to limit attempted source patterns during stochastic optimization. Clinical rules were designed through consultation with medical BT experts to identify source patterns that could potentially lead to poor implantations. Examples of hard-constraints included requiring a minimal $0.5 \mathrm{~cm}$ separation between intra-needle source placement to prevent dosimetric hot spots, or requiring a $0.5 \mathrm{~cm}$ needle gap near the urethral surface to minimize dose to the urethra (see Table 2.1 for a more comprehensive list). Finalized plans were exported to VariSeed 8.0 planning software (Varian Inc., Palo Alto, CA, USA) for final dosimetric evaluation. 
Table 2.1: Clinical rule strategies applied to stochastic optimization. The list is divided into "Never Do" and "Limits" components, both categories have exceptions. Low objective function values refer to values less than $5 \%$ of the initial objective function value.

Abbreviations: $\mathrm{N}=$ Needle, $\mathrm{S}=$ Source, $\mathrm{X}=$ "Cannot Be", $\mathrm{A}=$ "Always Must Be", Num $=$ Number, Pos $=$ Position, PTV $=$ Planning Target Volume, inf $=$ Inferior, $c c=$ cubic centimeter

\begin{tabular}{|c|c|c|c|c|}
\hline Type & $\begin{array}{l}\text { Applied } \\
\text { To }\end{array}$ & Rule & Range & Exception \\
\hline \multirow{6}{*}{$\begin{array}{l}\text { Never- } \\
\text { Do }\end{array}$} & $\mathrm{N}$ & $\overline{\mathrm{X}}$ & $<<$ Line 1 on template & None \\
\hline & $\mathrm{N}$ & $\mathrm{A}$ & $\begin{array}{l}\text { min. } 1 \text { axial prostate con- } \\
\text { tour }\end{array}$ & None \\
\hline & $\mathrm{N} / \mathrm{S}$ & $\mathrm{X}$ & $\begin{array}{l}\leq 0.5 \mathrm{~cm} \text { from } \mathrm{N} / \mathrm{S} \text { on axial } \\
\text { plane }\end{array}$ & None \\
\hline & S & $\mathrm{X}$ & $=1$ per any needle & None \\
\hline & $\mathrm{N} / \mathrm{S}$ & $\mathrm{X}$ & $\begin{array}{l}>1.5 \mathrm{~cm} \text { retraction from } \\
\text { base }\end{array}$ & None \\
\hline & $\mathrm{N}$ & $\mathrm{X}$ & $\begin{array}{l}\mathrm{c} 1, \mathrm{D} 1, \mathrm{~d} 1 \text { holes OR inf mid- } \\
\text { line holes }\end{array}$ & $\begin{array}{l}\text { low objective function: } \mathrm{Al}- \\
\text { low D-line needle }\end{array}$ \\
\hline \multirow[t]{6}{*}{ Limits } & $\overline{\mathrm{N}}$ & Num & $36-40$ & 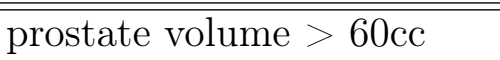 \\
\hline & $\mathrm{S}$ & Num & $<100$ & prostate volume $>60 \mathrm{cc}$ \\
\hline & $\mathrm{N}$ & Pos & $>1 \mathrm{~cm}$ to urethral surface & $\begin{array}{l}\text { low objective function: > } \\
0.5 \mathrm{~cm} \text { allowed }\end{array}$ \\
\hline & $\mathrm{N}$ & Pos & $\begin{array}{l}<0.5 \mathrm{~cm} \text { to PTV OR in di- } \\
\text { lated PTV margin }\end{array}$ & $\begin{array}{l}\text { low objective function: }< \\
0.8 \mathrm{~cm} \text { isotropic OR poste- } \\
\text { rior (to incl. seminal vesi- } \\
\text { cles) }\end{array}$ \\
\hline & $\mathrm{S}$ & Pos & $1 \mathrm{~cm}$ S-S spacing in needle & $\begin{array}{l}\text { low objective function: } \\
\text { apex }+1 \text {, base }-1 \text { allow } \\
0.5 \mathrm{~cm} \text { spacing in needle }\end{array}$ \\
\hline & $\mathrm{N} / \mathrm{S}$ & Pos & $\begin{array}{lll}\text { Fully load apex / base } \\
\text { planes with } \mathrm{N} / \mathrm{S}\end{array}$ & $\begin{array}{l}\text { Within limits of applied } \\
\text { rules }\end{array}$ \\
\hline
\end{tabular}


Table 2.2: Criteria used for pre-implantation treatment plan dosimetric evaluation; values are listed as a percentage of the prescribed monotherapy dose (145 Gy). VX\% = Volume of structure receiving at least $\mathrm{X} \%$ of prescription dose. DY\% $=$ Minimum dose to $\mathrm{Y} \%$ of structure volume. Abbreviations: PTV $=$ Planning Target Volume, $\mathrm{cc}=$ cubic centimeters

\begin{tabular}{||ccc||}
\hline Structure & Dose Metric & Criteria \\
\hline \hline Prostate & V $100 \%$ & $\geq 99 \%$ \\
\hline Prostate & V150\% & $\leq 60 \%$ \\
\hline Prostate & V $200 \%$ & $\leq 20 \%$ \\
\hline Prostate & D $90 \%$ & $113 \%-120 \%$ \\
\hline PTV & V100\% & $\geq 95 \%$ \\
\hline Urethra & D0.1cc & $<150 \%$ \\
\hline Rectum & D $1 \mathrm{cc}$ & $<100 \%$ \\
\hline
\end{tabular}

\subsubsection{Study Design}

A prospective study was conducted to compare the planning time of the ML algorithm to an expert RT planner, as well as IPSA-generated plans ( $n=10$ per group). Planning time was measured from deposition of the first source until the last plan modification using time points in the VariSeed planning system. A non-parametric, one-tailed Wilcoxon rank-sum test was use to compare the average planning times for each methodology $(\alpha=$ $0.05)$

Preoperative plan dosimetry was compared retrospectively between plans created by the ML algorithm, an expert RT planner, and a radiation oncologist trained in brachytherapy (BT) on a randomly selected set of 30 cases for each group. The RT plan is frequently modified following evaluation by a BT to yield a finalized plan. Dosimetry was evaluated using standard planning criteria for LDR (see Table 2.2) [111]. A multiple factor, oneway ANOVA ( $\alpha=0.05)$ was performed to evaluate differences between the three groups.

To evaluate the clinically subjective qualities of each plan a multi-institution, qualitative Likert scale questionnaire was designed to gauge 7 BT respondents' rankings of successive pairs of ML and RT plans. Fifteen LDR cases were evaluated with each case demonstrating an ML and RT plan pair; one control group (an IPSA plan generated by a novice planner) per respondent was also included. Three BTs had performed LDR cases for over 15 years, the remaining respondents had experience levels ranging from 1 year to 10 years. 


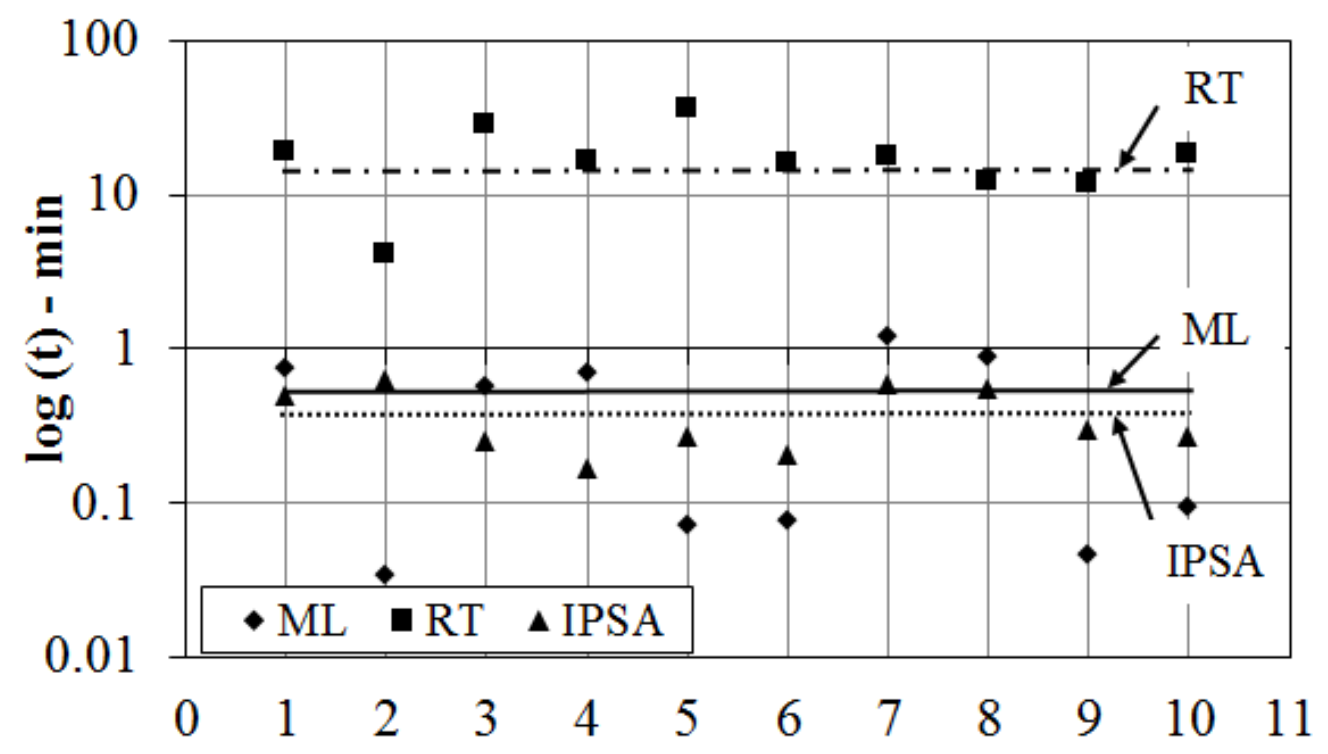

Figure 2.2: Comparison of treatment planning times. Treatment planning time for 10 patients planned using ML, RT, and IPSA methods displayed as a semi-log plot. The large variability in the ML group is evident. Horizontal bars represent the group means.

Brachytherapists evaluated 6 key measures of clinical quality: target coverage, normal tissue avoidance, confidence in their ability to implant the plan, modifications required to meet clinical standards if the plan was suboptimal, overall plan preference, and positive identification of the ML plan [112]. A multinomial ordered logistic regression was used to validate the effect of plan type (ML or RT) on respondent rankings, this was computed using SAS Analytics (SAS Institute, Cary, NC, USA) software.

\subsection{Results}

Planning time was significantly lower for ML $(0.84 \pm 0.57 \mathrm{~min})$ compared to manual RT (17.88 \pm 8.76 min) planners following a non-parametric Wilcoxon rank-sum test (p « 0.05); no significant difference was observed between the average ML and IPSA (0.37 \pm 0.17 min) plans. Fig 2.2 shows the difference in planning times between ML, RT, and IPSA groups for each patient.

No significant difference in prostate V100\%, V200\%, D90\%, PTV V100\%, urethra D 0.1cc and rectum D1cc metrics was observed following a one-way, multifactorial ANOVA $(\alpha=$ 0.05). A significant difference in the $\mathrm{V} 150 \%$ of the prostate was observed $(\mathrm{p}=0.002)$. A post-hoc test showed this difference was due a lower mean V150\% for the ML plans (52.9 

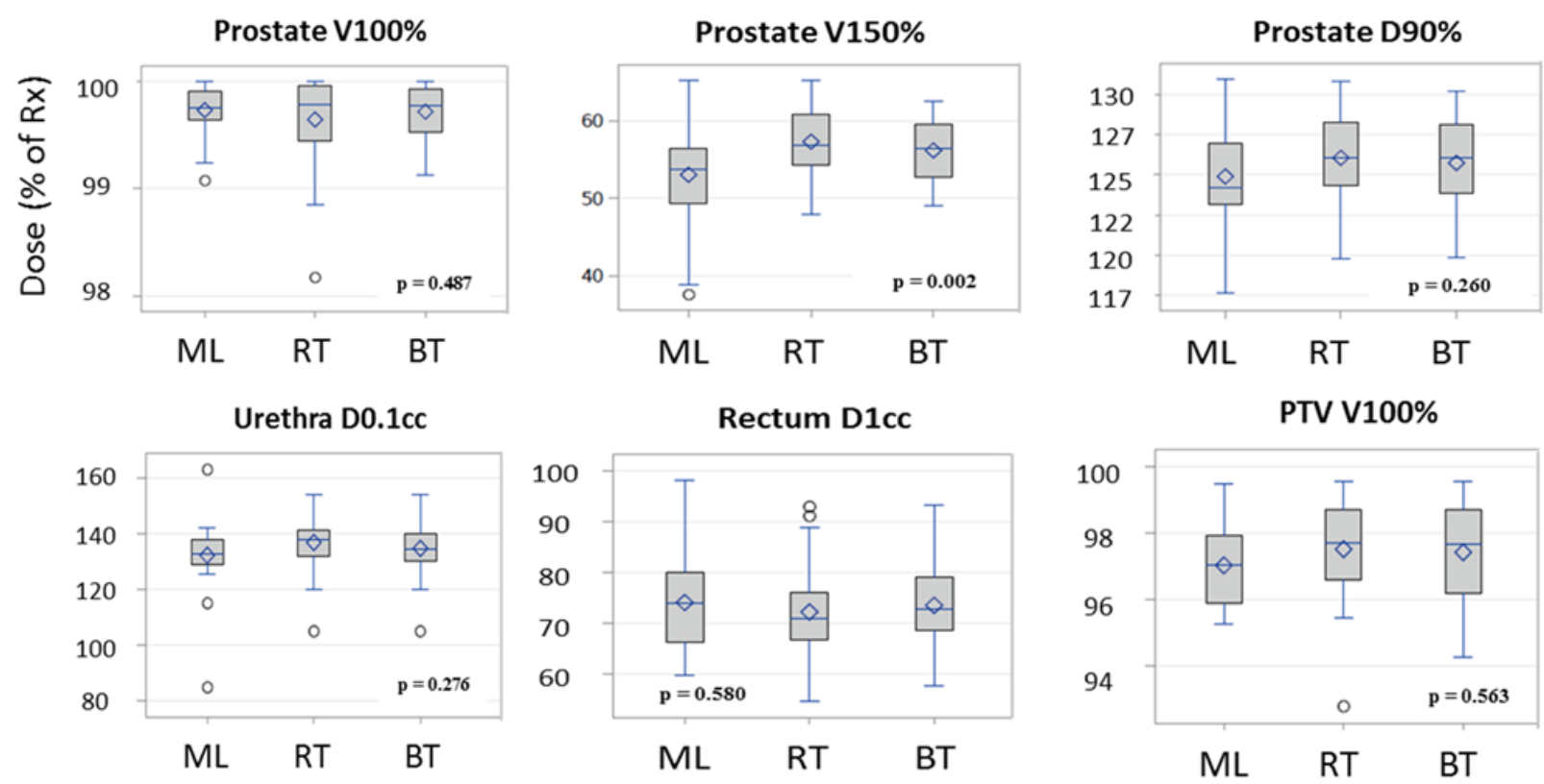

Figure 2.3: Pre-Implant Dosimetry.Comparison of the ML, RT, and BT plans for various quality metrics. Box-and-whisker plots showing group mean (diamond), median (horizontal bar), 1 standard deviation (box), the 95\% confidence intervals (whiskers), and relevant outliers (circles). Plotted with SAS University Edition 9.4 (SAS Institute, Cary, NC, USA). Abbreviations: $R x=$ Prescription Dose, PTV = Planning Target Volume

$\pm 6.3 \%)$ compared to RT $(57.3 \pm 4.4 \%)$, and MD (56.2 $\pm 3.7 \%)$ plans. Fig 2.3 outlines the results for the key dosimetric parameters used in this study.

Results of the qualitative ranking study by expert BTs are displayed in Fig 2.4. A small significant difference in respondent rankings for normal tissue avoidance, and the magnitude of plan modifications was observed. A BT respondent is 1.05 times $(\mathrm{OR}=$ 1.05, $\mathrm{p}=0.04)$ and 1.07 times $(\mathrm{OR}=1.07, \mathrm{p}=0.03)$, more likely to rank a plan as having " excellent sparing of normal tissues", and "requiring no further plan modifications" ( a 1 unit increase from 3 to 4) if it is an ML plan compared to a RT plan. The IPSA control plans performed relatively poorly in all categories evaluated. Respondents on average showed some difficulty in positively identifying the ML plans, although this improved with the number of years of experience of the respondent. Overall plan preference was marginally in favor of the ML-generated plans.

An illustrative example of how the ML algorithm may mimic features of expert treatment planners is shown in Fig 2.5; the inclusion of an IPSA generated plan is also included 


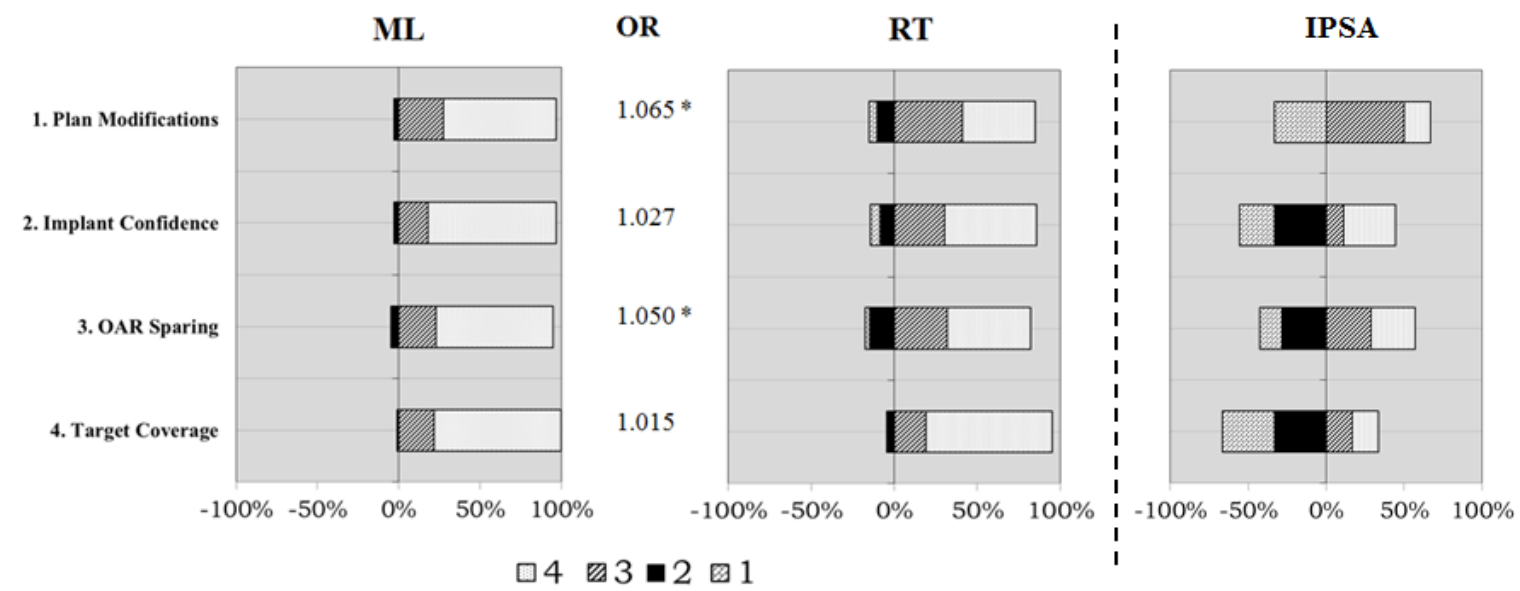

(a) BT respondent rankings for 4 plan categories comparing ML and RT plans; an IPSA plan was evaluated as a control for each respondent. The horizontal axis represents the normalized frequency of respondent answers: rankings of 4 and 3 are positive; rankings of 1 and 2 are negative. The log Odds Ratio (OR) is displayed in the central column, an asterisk is displayed beside significant values.

Categorical ranking: $4=$ Excellent, $3=$ Good, $2=$ Poor, $1=$ Very Poor.

Abbreviations: OAR $=$ Organ-At-Risk, $\mathrm{ML}=$ machine learning, $\mathrm{RT}=$ radiation therapist, $\mathrm{BT}$ $=$ brachytherapist, $\mathrm{OR}=$ Odds Ratio, IPSA $=$ Inverse-Planned Simulated Annealing

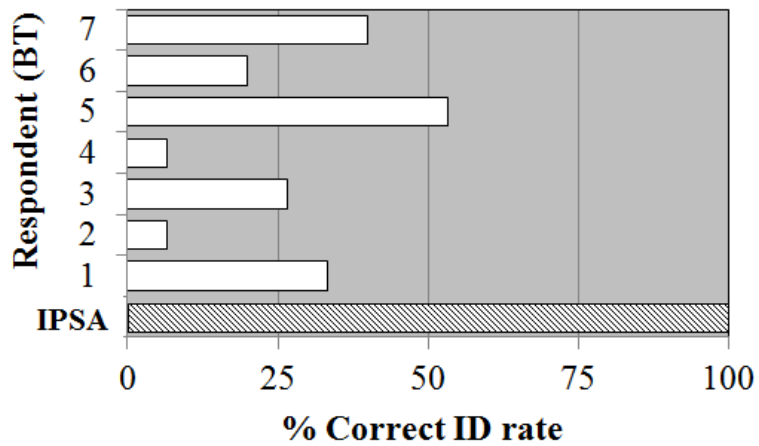

(b) Positive ID rate for BT respondents for ML plans. An IPSA control case was included.

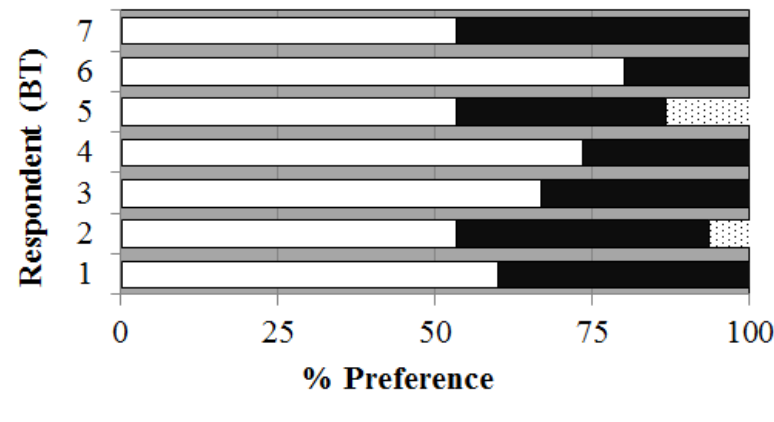

$\square$ Prefer ML $\square$ Prefer RT $\square$ Unsure / Equal Preference

(c) Normalized plan preference frequency stratified by BT respondent.

Figure 2.4: Results of the clinical qualitative studies 


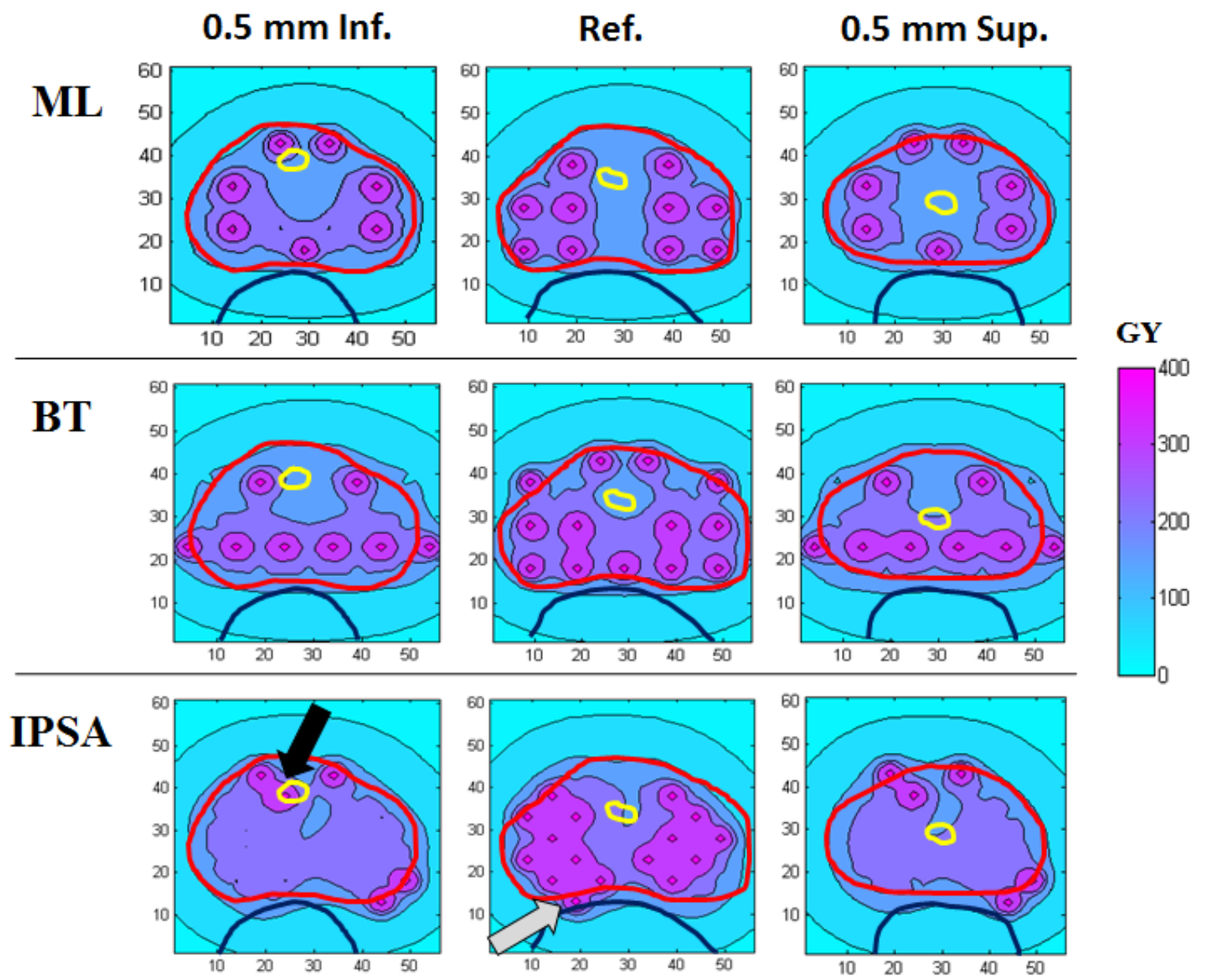

Figure 2.5: Comparison of treatment plans. Axial isodose representation of 3 sample treatment plans generated by an ML algorithm, a BT expert, and an IPSA algorithm (Varian VariSeed 8.0). The dark and light arrow show implant violations in the IPSA generated plan which prevent implantation, although these were likely to improve with expertise optimizing IPSA plans. Sources are represented as red diamonds. Contours include the prostate (red), urethra (yellow), and rectum (dark blue). The $\mathrm{x}$ - and $\mathrm{y}$ axis dimensions are in millimeters.Abbreviations: Ref $=$ Reference Plan, IPSA $=$ Inverse Planned Simulated Annealing, Gy = Gray, Inf. = Inferior, Sup. = Superior 
for comparison to a generalized stochastic approach. Qualitative plan features indicative of improved implantation were highly similar between ML and BT groups.

\subsection{Discussion}

With the exception of the prostate V150\% the ML-generated dosimetry metrics were equivalent to those of the RT and BT plans; although statistically significant the V150\% was lower by an average of only $4 \%$ from the expert-planned cases and therefore not clinically significant. Planning time was significantly different with the ML plans taking on average $<1$ minute to create clinically adequate solutions. Despite the added computational complexity of an ML approach the average planning time was highly comparable to IPSA-generated plans [76], both of which are considerably faster than manual RT planning approaches. Recent studies predict that the incidence rate of prostate cancer may nearly double in the next 15 years due to an aging population [2], if this forecast becomes a reality, burdened brachytherapy departments may simply be unable to accommodate the increased patient volumes purely due to time constraints. The incorporation of an ML algorithm in the brachytherapy workflow is expected to offer time and resource savings in this regard.

This study sought to evaluate clinical implant features not captured by dosimetric analysis alone; overall, the clinical quality as evaluated by BT respondents was equivalent between ML and RT groups. The target coverage and implant confidence rankings were essentially identical between the ML and RT groups. The differences in the "OAR sparing" and "plan modification" categories indicated that BT respondents were more likely to report a singleunit improvement (a ranking of 4 compared to 3, for example) in these categories if a plan was generated by ML compared to an RT planner. This small difference may indicate a lack of uniform plan quality among manually created RT plans, even within a single institution. The ML brachytherapy workflow may therefore offer improved uniformity and standardization in the creation of high quality plans compared to multiple human planners with varying levels of expertise. Although a small sample size was used and planners were 
relatively inexperienced with IPSA workflows many of the control cases demonstrated noticeably worse qualitative rankings. In particular confidence in the implant was ranked worse than either ML or RT plans and despite passing dose metrics the qualitative target coverage (i.e. the relative position of the isodose lines) was also generally very poor for these plans. Additionally, the results of this clinical study indicate that plans can be generated using ML that mimic several features of expert BT planners, as corroborated by the difficulty among BT respondents in differentiating blinded ML plans; by comparison, BT respondents were able to correctly identify the IPSA control case $100 \%$ of the time.

Incorporation of a training database of prior knowledge into the LDR brachytherapy planning workflow, in addition to implementing clinical rule restrictions during the optimization process, incorporates a measure of radiation oncologist technique and skill into the planning process, one of the direct benefits of ML approaches over naive stochastic optimization [112]. It remains to be seen if improvements in plan standardization translate to improved side-effects or long-term outcomes. Similar ML methods have been implemented in radiological imaging diagnosis which not only helped reduce staff workload but improve standardization of diagnosis both within and across centers [84,85]. For centers with limited brachytherapy experience (or limited annual patient volumes) an ML-based approach, which constrains plans to follow clinical rules could additionally offer a means to reduce the risk of errors being propagated during planning.

Limitations of un-supervised ML approaches result from the need for restrictively large training databases of prior information for insight and model development [113]. Excessively sparse, redundant, or over-fitted data may produce poor results and puts significant emphasis on maintaining data quality. Similarly, poorly descriptive features, or excessive optimization of parameters may cause under- or over-generalization of the ML model. These limitations were not present in the supervised ML approach taken in this study. The LDR training data was filtered according to post-operative dosimetric outcomes and was used to maintain consistent data quality throughout; subsequently, feature extraction methods were non-random and targeted to specific clinical features deemed important for implantation by expert BT physicians. Poor generalization of the algorithm was signif- 
icantly limited through clinical rule implementation during stochastic optimization; this functioned as a form of regularization to improve model fitting. Regularization, a method to reduce the detrimental effects of poor data modelling, could further improve the generalizability of the ML algorithm and is left to future studies to adapt differing models [114]. Although the training database was based on a single center experience the qualitative study evaluated responses from 2 respondents at an external international brachytherapy program; no significant differences in rankings were observed between respondents indicating that the current ML model may be applicable to centers with widely differing protocols.

Further development of the core algorithm would adapt this framework for intraoperative LDR planning, and for High-Dose-Rate (HDR) brachytherapy which is increasing in popularity as a method to deliver hypofractionated doses due to its improved dose modulation $[57,115]$. Prostate HDR brachytherapy may offer an alternate application of the ML algorithm.

\subsection{Conclusion}

In contrast to traditional algorithmic planning methods the experience of expert BTs and treatment planners is incorporated within ML approaches. Dosimetrically optimal plans with higher chances of being successfully implanted in the patient are possible, in significantly shorter timeframes. It is expected that further validation of the algorithm components and incorporation of more immediate feedback of post-implant results may improve the adaptive ability of the ML algorithm. 


\section{Chapter 3}

\section{Conclusion}

\subsection{Thesis Summary}

The primary objective of this thesis was to demonstrate that supervised and highly focused Machine Learning (ML) algorithms could be used to generate Low-Dose-Rate (LDR) brachytherapy plans of equal or superior quality to expert brachytherapists (BT). Chapter 1 introduced the developing problem of up to a $97 \%$ increase in prostate cancer incidence in the next 15 years [2]; this extrapolated trend highlighted the urgent need for high quality, effective, and efficient therapy for patients with prostate cancer. Brachytherapy was explored as one of the most effective methods for treating localized disease with over $90 \%$ long-term disease control rates and tolerable side-effects $[31,42,46]$. The use of computer optimization to accurately plan source patterns in prostate brachytherapy has seen considerable resurgence in recent years [70]. Stochastic optimization algorithms, although capable of rapid dosimetric optimization, regularly fail to take into account the actual ability of BTs to recreate the plan at the time of implantation [72]. To this end chapter 2 explored machine learning frameworks, which rely on databases of previous knowledge acquired from years of experience, to offer a method of improved planning in brachytherapy.

Chapter 2 presented the results from a clinical evaluation of an ML algorithm specifically 
adapted to perform prostate LDR brachytherapy planning. The aim of this evaluation was to show how an ML framework can be used to generate plans that mimic those created manually by experts [76]. The planning efficiency, dosimetry, and clinical quality of these plans were compared to similar plans performed by experts. This study showed that planning time was significantly shorter for ML plans at an average of $0.84 \pm 0.57$ minutes while expert plans had not only more variance in planning time but on average took 17.88 \pm 8.76 minutes to generate plans of equal quality. Planning time for IPSA cases was shorter than both ML and RT plans at $0.37 \pm 0.17$ minutes; the ML and RT planning time was not significantly different indicating comparable planning time among algorithms. Plan dosimetry was comparable between ML plans, an expert radiation therapist (RT), and the final accepted BT plan with only a lower prostate V150\% in the ML plans; however, the decrease was small and likely to be insignificant clinically. The majority of studies published on new optimization algorithms frequently evaluate dosimetry alone as measures of plan success, this alone may be insufficient to gauge plan quality. In addition to dosimetry this thesis evaluated expert BT critique of each plan across six qualitative categories. This study demonstrated a statistically significant log Odds Ratio (OR) benefit in the "OAR sparing" and "plan modifications" categories (following an ordered logistic regression), as respondents selected ML over RT plans. Although this was statistically significant the OR was small and in essence the evaluated clinical quality was nearly identical. By comparison the IPSA control cases performed poorly compared to both ML and RT groups; in particular the qualitative target coverage was ranked very poor for most cases. This poor quality may also be partially attributable to the relative inexperience of planners with IPSA optimization. A benefit of the ML approach was that the majority of respondent BTs were unable to differentiate expert plans from those created by an ML algorithm, corroborating the hypothesis that ML can be used to generate plans features which mimic those of a human planner; the IPSA control was identified as such by all BT respondents. This type of qualitative evaluation although a necessity for finalizing treatment plans is rarely performed in analyses of optimization algorithms and may become a new standard in brachytherapy treatment planning. 
This thesis was able to demonstrate that ML brachytherapy treatment plans were equivalent to those generated by experts in terms of plan dosimetry and clinical quality. Several advantages were also evident for ML over the conventional manual planning approach as well as optimization algorithms used in brachytherapy planning. It follows that with a reduced planning time significant improvements in clinical workflow efficiency may be realized as fewer staff resources are required for plan creation. The short planning time also allows adaptation of the algorithm for the more time-sensitive intraoperative brachytherapy workflow. Improvements in plan quality and homogeneity are also expected benefits of ML implementation, especially compared to conventional stochastic algorithms where widely differing plan results are produced upon optimization, many of which are not clinically feasible. The homogeneity in treatment plan quality afforded by ML offers a more consistent and long-term approach to modulate LDR brachytherapy quality than the educational training programs used currently, both across and between centers with differing protocols. By offering more clinically feasible, evidence-based treatment plans it is hoped that the number of sub-standard implants will decrease following more widespread adoption of ML workflows, particularly for centers with reduced patient volumes. Further study into the nature of widespread adoption of an ML workflow within the brachytherapy community is warranted.

\subsection{Discussion \& Future Work}

\subsubsection{Further Optimization of the ML algorithm}

Although successfully implemented within the LDR brachytherapy planning framework the ML algorithm designed in this thesis can be improved by enhancing its performance and improving its generalization to more outlying prostate cases. The following components could potentially be modified:

\section{Database \& Feature Extraction}

The science behind database and retrieval optimization - specifically when the data 
is related medical data - is a field in its infancy [116,117]. Further studies need to be conducted into the appropriate training database size for brachytherapy-specific applications. Excessively large database sizes may slow down performance or contain redundant information, while databases of insufficient size may produce solutions which are insufficiently generalized [117]. Similarly, feature extraction in the ML model employed by this thesis is an expert-driven process requiring feedback from clinician experts to determine viable features, the number and quality of features required for optimal performance is still an area of active exploration [118]. There has been some interest in using meta-data - the patient's staging results, dosimetry, PSA results, genetics etc. - to supplement information provided by anatomical contour information [119]. This may provide a further method of discriminating treatment plans than geometry alone and would be worthy of examination for the ML implementation used in this thesis. With improved discrimination of the prostate gland and OARs direct feature extraction from imaging alone may help supplant the contour feature extraction method used in the current implementation. Several centers are utilizing Magnetic Resonance Imaging (MRI) which has superior soft-tissue visualization of the prostate gland; the considerable imaging detail afforded by MRI may serve to inform yet undiscovered key features to differentiate between prostates [120,121]. Extracting key imaging features using image processing techniques alone has been employed in convolutional neural networks for feature extraction and would be of interest to explore for the brachytherapy-specific ML algorithm evaluated in this thesis [122]. Supervised ML algorithms for auto-contouring of the prostate gland on Transrectal Ultrasound (TRUS) imaging alone have also been evaluated with excellent results [123]. Incorporation of these auto-contouring algorithms with the planning ML algorithm presented in this thesis is readily achievable given the available training data, it is hoped that this will further decrease the reliance on expertise in the planning process.

\section{Optimization \& Parameter Tuning}

The IPSA algorithm was used in this thesis due to its success in optimizing dosimetry in LDR brachytherapy. While this was appropriate when starting from randomly selected source patterns when starting with a source pattern that is already near a viable solution 
for the specific prostate geometry this may not be the most effective option. Several machine learning algorithms use gradient descent approaches to speed up the optimization process; this approach generally follows the fastest gradient regardless of any large local minima [124]. It can be hypothesized that starting optimization near a local viable solution may be sufficient when employed in the context of the ML algorithm in this thesis. Many other algorithms also use this methodology to optimize various algorithmic parameters, a process in this thesis that was done by cross-validation [125]. This gradient descent approach will be examined in future renditions of the ML algorithm for optimization of various parameters and as a method to replace the current IPSA algorithm. Gradient descent methods could also be employed in selecting and fine-tuning optimal combinations of clinical rules to implement for different prostate geometries, rule violations in a specified prostate shape may not be applicable for all geometries, or even at all instances in the optimization of the source pattern. By applying gradient descent to not only source pattern optimization but to clinical rule optimization a method of fine-tuning these rules can be developed for specific geometries.

\section{"Learning" Framework}

The current iteration of ML adaptation uses post-operative dosimetric results to filter which LDR plans are appended to the training dataset and which are discarded. In this manner only successful plans are "learned" while plans that fared poorly are not retained. This feedback mechanism is exceedingly slow due to the large delay between implantation and post-operative follow-up (approximately 1 month). Future developments will include updating the speed and quality of this feedback mechanism by finding intermediate points of evaluation between pre-implant plans and post-operative dosimetry. Evaluative studies need to be undertaken comparing treatment plan quality (as well as treatment outcomes) when including intermediate time points; inclusion of these intermediate time points may potentially degrade plan quality correlation with post-operative dosimetry but may also improve the algorithmic learning speed significantly. The inclusion of different evaluating time points in the workflow therefore needs to be carefully evaluated for impacts on plan and treatment outcomes before implementation. It has also been observed that 
needles used to implant sources frequently deflect and (along with post-implant edema) cause the source pattern to diverge from the original treatment plan [126,127]. In future implementations the dynamics of the source migration caused by these two phenomena will be examined. Models for source migration could be implemented in the ML algorithm to further decrease the risk of poorly recreating the treatment plan. Finally, although many of these methods can be attempted with some success the inherent delay in the feedback of quality treatment outcomes data may simply be a limitation of the LDR brachytherapy workflow.

\subsubsection{Machine Learning for HDR Brachytherapy}

High-Dose-Rate (HDR) brachytherapy is a more recent modality for delivering brachytherapy to patients. Instead of permanently implanted, low-dose sources, HDR delivers high doses of radiation to the prostate gland in a short time frame through the temporary placement of a single, high activity radioactive source [128]. Several catheters are temporarily implanted into the prostate through which an Iridium-192 or other high activity source is sequentially introduced. The dwell times and positions of the source are optimized to maximize the dose to the prostate and minimize dose to the OARs [127]. Although more radiation safety considerations are needed due to the high source activity HDR brachytherapy may offer better dose modulation over LDR brachytherapy as shown in Morton et al. [57]; it is quickly being adopted as a treatment of choice for many centers. Patients also do not require post-operative dosimetry at 1 month follow-ups as this information can be obtained immediately after the treatment.

The findings of Chapter 2 demonstrated that it may become possible to apply ML to HDR brachytherapy treatment planning, the ML algorithm used in this thesis may actually be more appropriate for HDR than in LDR brachytherapy. The versatility of the ML method to include any contour or imaging information and extract primitive features has wide application in HDR brachytherapy for both prostate and gynecological cancers. HDR brachytherapy has greater number and variability in catheter and source positions than LDR, has significantly shorter time frames for planning as it is typically 
done in real-time, and specifically in gynecological brachytherapy there is considerably more variation in target and normal tissue contours [128]. These more complex features make HDR brachytherapy a prime application for ML in the near future with the modality benefitting significantly from ML. In addition, post-operative dosimetry is available immediately following treatment and may significantly improve the adaptation speed of an HDR ML algorithm. It is hoped that ML frameworks will become a new standard to adapt brachytherapy cases for different cancer sites and offer a means of improving standardization of brachytherapy.

\subsubsection{Machine Learning for Prostate Radiotherapy}

Several other potential applications exist for Machine Learning algorithms in prostate radiotherapy that are unrelated to brachytherapy alone. Although these algorithms are primarily investigational they hold significant promise in improving the speed, accuracy, and efficiency of prostate radiotherapy. ML algorithms for radiological or histological diagnosis of prostate cancer have been discussed in earlier chapters which predict the presence of prostate cancer based on radiological and histopathological imaging features [129]. Multicenter learning for prostate treatment plan creation (for both external radiotherapy and brachytherapy) is a possibility; anonymized meta-data may be transferrable between centers and offers an indirect method of sharing expertise, or even aggregating data for predicting outcomes more accurately [130,131]. More common applications that focus on workflow efficiencies include methods for automated contouring of target structures such as the prostate gland [123,132], and treatment planning assistance tools in Intensity Modulated Radiotherapy (IMRT) [133]. An excellent book by El Naqa et al. (2015) highlights many of these emerging applications for radiotherapy in more detail and outlines the considerable room for growth that exists in this field [134]. Future studies will examine the combination and implementation of many of these ML paradigms to improve the efficiency of the prostate radiotherapy workflow in addition to brachytherapy. 


\section{Bibliography}

[1] Statistics Canada. 2015. CANSIM table 102-0561 - Leading Causes of Death, Total Population, by Age Group and Sex, Annual. CANSIM database, Health Canada.

[2] Canadian Cancer Statistics 2015. Toronto, ON; Canadian Cancer Society

[3] Tindall EA, Bornman MSR, Zyl SV, et al. 2013. Addressing the contribution of previously described genetic and epidemiological risk factors associated with increased prostate cancer risk and aggressive disease within men from South Africa. BMC Urology; 13: 74

[4] Muller DC, Severi G, Baglietto L, et al. 2009. Dietary patterns and prostate cancer risk. Cancer Epidemiol Biomarkers Prev; 18(11): 3126-9

[5] Bloom JR, Steward SL, Oakley-Girvans I, et al. 2006. Family history, perceived risk, and prostate cancer screening among African American men. Cancer Epidemiol Biomarkers Prev; 15(11): 2167-73

[6] Thompson IM, Ankerst AP, Chi C, et al. 2006. Assessing prostate cancer risk: results from the prostate cancer prevention trial. J Natl Cancer Inst; 98(8): 529-34

[7] Cooperberg MR, Lubeck DP, Meng MV, et al. 2004. The changing face of low-risk prostate cancer: trends in clinical presentation and primary management. J Clin Oncol; 11: 2141-9

[8] Greenhalgh R, Kirby RS. 2002. Anatomy and physiology of the prostate and benign prostatic hyperplasia. Atlas Urol Clin; 10: 1-9

[9] Lija H. 2003. Biology of prostate-specific antigen. Urol; 62(5S1): 27-33

[10] Diamandis EP. 2000. Prostate-specific antigen: a cancer fighter and a valuable messenger? Clin Chem; 46(7): 896-900

[11] Benedettini E, Nguyen P, Loda M. 2008. The pathogenesis of prostate cancer: from molecular to metabolic alterations. Diagn Histopath; 14(5): 195-201

[12] Constantin TV, Madan VL, Constantin MM, et al. 2014. The role of prostatespecific antigen in prostate cancer screening. Revista Romana de Medicina de 
Laborator; 22(2): 275-81

[13] Kim EH, Andriole GL. 2015. Prostate-specific antigen-based screening: controversy and guidelines. BMC Medicine; 13: 62-6

[14] Hoffman RM, Zeliadt SB. 2010. The cautionary tale of PSA testing: comment on 'Risk profiles and treatment patterns among men diagnosed as having prostate cancer and prostate-specific antigen level below $4.0 \mathrm{~nm} / \mathrm{mL}$ '. Archives of Internal Medicine; 179: 1262-3

[15] Ankerst DP, Thomson IM. 2006. Sensitivity and specificity of prostate-specific antigen for prostate cancer detection with high rates of biopsy verification. Archivio Italiano di Urologica e Andrologica; 78(4): 125-9

[16]. Sandhu GS, Andriole GL. 2012. Overdiagnosis of prostate cancer. J Natl Cancer Inst Monogr;45:146-51

[17]. Andriole GL, Crawford ED, Grubb RL, Buys SS, Chia D, Church TR, et al. 2012. Prostate cancer screening in the randomized prostate, lung, colorectal, and ovarian cancer screening trial: Mortality results after 13 years of follow-up. J Natl Can Inst;104(2):125-32

[18]. Zhu X, Van Leeuwen PJ, Bul M, Otto SJ, de Koning HJ, Bangma CH, et al. 2011. Disease-specific survival of men with prostate cancer detected during the screening interval: results of the European randomized study of screening for prostate cancer-Rotterdam after 11 years of follow-up. Eur Urol; 60(2):330-6

[19]. Schroeder FH, Hugosson J, Roobol MJ, Tammela TL, Ciatto S, Nelen V, et al. 2012. Prostate-cancer mortality at 11 years of follow-up. N Engl J Med; 366(11): 981-90

[20] Hayes JH, Barry MJ. 2014. Screening for prostate cancer with the prostatespecific antigen test: A review of current evidence. JAMA;311(11):1143-9

[21] Mohler JL, Armstron AJ, Bahnson RR, et al. 2016. Prostate Cancer, Version 1.2016. J Natl Compr Canc Netw; 14: 19-30

[22] Humphrey PA. 2004. Gleason grading and prognostic factors factors in carcinoma of the prostate. Modern Pathology; 17: 292-306

[23] Draisma G, Etzioni R, Tsodikoy A. 2009. Lead time and overdiagnosis in prostate-specific antigen screening: importance of methods and context. J Natl Cancer Inst; 101: 374-383

[24] Wilt TJ. 2002. Clarifying uncertainty regarding detection and treatment of early-stage prostate cancer. Seminars in Urologic Oncology; 20(1): 10-7

[25] Keyes M, Crook J, Morton G, et al. 2013. Treatment options for localized 
prostate cancer. Can Fam Phys; 59(12): 1269-74

[26] Klotz LH, Zhang L, Lam A, et al. 2010. Clinical results of long-term follow-up of a large, active surveillance cohort with localized prostate cancer. Am Soc Clin Oncol; 28(1): 126-31

[27] Mazur DJ, Hickam DH. 1996. Patient preferences for management of localized prostate cancer. West J Med; 165(1-2): 26-30

[28] Chapple A, Ziebland S, Herxheimer A, et al. 2002. Is "watchful waiting" a real choice for men with prostate cancer? A qualitative study. BJUI; 90(3): 257-64

[29] Suardi N, Capitanio U, Chun FK, et al. 2008. Currently used criteria for active surveillance in men with low-risk prostate cancer: an analysis of pathologic features. Cancer; 113(8): 2068-72

[30] Penson DF. 2009. Active surveillance: not your father's watchful waiting. Oncology; 23(11): 980-81

[31] Grimm P, Billiet I, Bostwick D, et al. 2012. Comparative analysis of prostatespecific antigen free survival outcomes for low, intermediate, and high risk prostate cancer treatment by radical therapy: Results from the Prostate Cancer Results Study Group. BJU Int; 109(1): 22-9

[32] Bianco FJ, Riedel ER, Begg CB, et al. 2005. Variations among high volume surgeons in the rate of complications after radical prostatectomy: further evidence that technique matters. J Urol; 173(6): 2099-103

[33] Walsh PC, Marschke P, Ricker D, et al. 2000. Patient-reported urinary incontinence and sexual function after anatomic radical prostatectomy. Urol; 55(1): $58-61$

[34] Stanford JL, Feng Z, Hamilton AS, et al. 2000. Urinary and sexual function after radical prostatectomy for clinically localized prostate cancer: the Prostate Cancer Outcomes study. JAMA; 283(3): 354-60

[35] Alemozaffar M, Regan MM, Cooperberg MR, et al. 2011. Prediction of erectile function following treatment for prostate cancer. JAMA; 306(11): 1205-14

[36] Crook J. 2015. Long-term oncologic outcomes of radical prostatectomy compared with brachytherapy based approaches for intermediate- and high-risk prostate cancer. Brachytherapy; 14(2): 142-7

[37] Ritter M. 2008. Rationale, conduct, and outcome using hypofractionated radiotherapy in prostate cancer. Semin Radiat Oncol; 18(4): 249-256

[38] Hall EJ, Brenner DJ. 1991. The dose-rate effect revisited: radiobiological considerations of importance in radiotherapy. Int J Radiat Oncol Biol Phys; 21(6): 
[39] Pickles T, Keyes M, Morris WJ. 2010. Brachytherapy or conformal external radiotherapy for prostate cancer: a single-institution matched-pair analysis. Int J Radiat Oncol Biol Phys; 76(1): 43-9

[40] Yu Y, Anderson LL, Li Z, et al. 1999. Permanent prostate seed implant brachytherapy: report of the American Association of Physicists in Medicine Task Group No. 64. Med Phys; 26(10): 2054-76

[41] Kubo HD, Glasgow GP, Pethel TD, et al. 1998. High dose-rate brachytherapy treatment delivery: report of the AAPM Radiation Therapy Committee Task Group No. 59. Med Phys; 25(4): 375-403

[42] Keyes M, Miller S, Moravan V, et al. 2009. Predictive factors for acute and late urinary toxicity after permanent prostate brachytherapy: long-term outcome in 712 consecutive patients. Int J Radiat Oncol Biol Phys; 73(4): 1023-32

[43]. Morris WJ, Keyes M, Palma D, et al. 2009. Population-based study of biochemical and survival outcomes after permanent 125I brachytherapy for low- and intermediate-risk prostate cancer. Urology; 73(4): 860-7

[44]. Crook J, Borg J, Evans A, et al. 2011. 10-year experience with I-125 prostate brachytherapy at the Princess Margaret Hospital: results for 1,100 patients. Int J Radiat Oncol Biol Phys; 80(5): 1323-9

[45]. Zebentout O, Apardian R, Beaulieu L, et al. 2010. Clinical outcome of intermediate risk prostate cancer treated with iodine 125 monotherapy: The Hotel-dieu of Quebec experience. Cancer Radiother; 14(3): 183-8

[46] Cesaretti JA, Kao J, Stone NN, et al. 2007. Effect of low dose-rate prostate brachytherapy on the sexual health of men with optimal sexual function before treatment: analysis at $\geq 7$ years of follow-up. BJU Int; 100(2): 362-7

[47] Giberti C, Chino L, Gallo F, et al. 2009. Radical retropubic prostatectomy versus brachytherapy for low-risk prostatic cancer: a prospective study. World J Urol; 27(5): $607-12$

[48] Crook MJ, Gomez-Iturriaga A, Wallace K, et al. 2011. Comparison of healthrelated quality of life 5 years after SPIRIT: surgical prostatectomy versus interstitial radiation intervention trial. J Clin Oncol; 29(4): 362-8

[49] Stock RG, Stone NN. 2002. Importance of post-implant dosimetry in permanent prostate brachytherapy. Eur Urol; 41: 434-9

[50] Prestidge BR, Bice WS, Kiefer EJ, et al. 1998. Timing of computed tomographybased post-implant assessment following permanent transperineal prostate brachytherapy. Int J Radiat Oncol Biol Phys; 40: 1111-5 
[51] Rustgi SN. 1992. Photon spectral characteristics of a new double-walled Iodine 125 source. Med Phys; 19(4): 927-31

[52] Rivard MJ, Coursey BM, DeWard LA, et al. 2004. Update of AAPM Task Group No. 43 Report: a revised AAPM protocol for brachytherapy dose calculations. Med Phys; 31(3): 633-674

[53] Nag S, Bice W, De Wyngaert K, et al. 1995. American Brachytherapy guidelines for post-implant dosimetry for prostate brachytherapy. Int J Radiat Oncol Biol Phys; 46: $221-30$

[54] Stock RG, Stone NN, Tabert A, et al. 1998. A dose response study for I125 prostate implants. Int J Radiat Oncol Biol Phys; 41: 101-8

[55] Vijverberg PL, Blank LE, Dabhoiwala NF, et al. 1993. Analysis of biopsy findings and implant quality following ultrasonically-guided 125I implantation for localized prostatic carcinoma. BJU; 72(4): 470-7

[56] Veccia A, Caffo O, Fellin G, et al. 2015. Impact of post-implant dosimetric parameters on the quality of life of patients treated with low-dose rate brachytherapy for localized prostate cancer: results of a single-institution. Radiat Oncol; 10: 130-8

[57] Morton G, Hoskin PJ. 2013. Brachytherapy: current status and future strategies - can High Dose Rate replace Low Dose Rate and external beam radiotherapy? Clin Oncol; 25(8): 474-82

[58] Wang Y, Sankreacha R, Al-Hebshi A, Loblaw A, Morton G. 2006. Comparative study of dosimetry between high-dose-rate and permanent prostate implant brachytherapies in patients with prostate adenocarcinoma. Brachytherapy; 5: 251-255

[59] Le-Fur E, Malhaire JP, Baverez D, et al. 2012. Impact of learning curve and technical changes on dosimetry in low-dose brachytherapy for prostate cancer. Strahlenther Onkol; 188: 1091-5

[60] Keyes M, Schellenberg D, Moravan V, et al. 2006. Decline in urinary retention incidence in 805 patients after prostate brachytherapy: the effect of learning curve. Int J Radiat Oncol Biol Phys; 64: 825-34

[61] Murakami N, Itami J, Okuma K, et al. 2008. Urethral dose and increment of international prostate symptom score (IPSS) in transperineal permanent interstitial implant (TPI) of prostate cancer; Strahlenther Onkol; 184: 515-9

[62] Liu HW, Malkoske K, Sasaki D, et al. 2010. The dosimetric quality of brachytherapy implants in patients with small prostate volumes depends on the experience of the brachytherapy team. Brachytherapy; 9: 202-7

[63] Hughes L, Waterman FM, Dicker AP. 2005. Salvage of suboptimal prostate 
seed implantation: reimplantation of underdosed region of prostate base. Brachytherapy; 4(2): 163-70

[64] Sasaki H, Kido M, Miki K, Kuruma H, Takahashi H, Aoki M, et al. 2014. Salvage partial brachytherapy for prostate cancer recurrence after primary brachytherapy. Urology; 21(6): 572-77

[65] Sylvester JE, Grimm PD, Wong J, et al. 2011. Fifteen-year biochemical relapse-free survival, cause-specific, and overall survival following I(125) prostate brachytherapy in clinically localized prostate cancer: Seattle experience. Int J Radiat Oncol Biol Phys; 81: 376-381

[66] Nasser NJ, Sappiatzer J, Wang Y, Borg J, Saibishkumar EP. 2015. Dosimetric evaluation of clinical target volume in the postimplant analysis of low dose rate brachytherapy for prostate cancer. Brachytherapy; 14(2): 189-96

[67] Hamacher HW, Kufer KH. 2002. Inverse radiation therapy planning - a multi objective optimization approach. Discrete Applied Mathematics; 118(1-2): $145-61$

[68] Lu JY, Zhang JY, Li M, et al. 2015. A simple optimization approach for improving target dose homogeneity in intensity-modulated radiation therapy for sinonasal cancer. Scientific Reports; 5: 153-61

[69] Smith WP, Kim M, Holdsworth C, et al. 2016. Personalized treatment planning with a model of radiation therapy outcomes for use in multiobjective optimization of IMRT plans for prostate cancer. Radiat Oncol; 11: 38

[70] Dinkla AM, Van der Laarse R, Kaljouw E, Pieters BR, Koedooder K, Van Wieringen N, et al. 2015. A comparison of inverse optimization algorithms for HDR / PDR prostate brachytherapy treatment planning. Brachytherapy; 14(2): 279-88

[71] Pouliot J, Tremblay D, Roy J, Filice S. 1996. Optimization of permanent 125I prostate implants using fast simulated annealing. Int J Radiat Oncol Biol Phys; 36(3): $711-20$

[72] Lessard E, Pouliot J. 2001. Inverse planning anatomy-based dose optimization for HDR brachytherapy of the prostate using fast simulated annealing algorithm and dedicated objective function. Med Phys; 28(5): 773-9

[73] Lessard E, Hsu IC, Pouliot J. 2007. Clinical benefits of inverse planning for high dose rate brachytherapy. IFMBE Proceedings; 14(3): 1730-4

[74] Pokharel S, Rana S, Blikenstaff J, et al. 2013. Evaluation of hybrid inverse planning and optimization (HIPO) algorithm for optimization in real-time, high-doserate (HDR) brachytherapy for prostate. J Appl Clin Med Phys; 14(4): 4198

[75] Gorissen BL, Hertog DD, Hoffmann AL. 2013. Mixed integer programming 
improves comprehensibility and plan quality in inverse optimization of prostate HDR brachytherapy. Phys Med Biol; 58(4): 1041-57

[76] Lahanas M, Baltas D, Zamboglou N. 2003. A hybrid evolutionary algorithm for multi-objective anatomy-based dose optimization in high-dose-rate brachytherapy. Phys Med Biol; 48(3): 399-415

[77] Yu Y, Schell MC. 1996. A genetic algorithm for the optimization of prostate implants. Med Phys; 23: 2085 - 91

[78] Kirkpatrick S, Gelatt CD, Vecchi MP. 1983. Optimization by simulated annealing. Science; 220(4598): 671-680

[79] Yu Y, Zhang JB, Cheng G, Schell MC, Okunieff P. 2000. Multi-objective optimization in radiotherapy: applications to stereotactic radiosurgery and prostate brachytherapy. Artif Intell Med; 19(1): 39-51

[80] Han BH, Wallner K, Merrick G, Butler W, Sutlief S, Sylvester J. 2003. Prostate brachytherapy seed identification on post-implant TRUS images. Med Phys; 30(5): 898-900

[81] Hemanth DJ, Anitha J. Chapter 12: Computational intelligent techniques for pattern recognition in biomedical image processing applications. In: Machine Learning Algorithms for Problem Solving in Computational Applications. IGI Global, 2012:195-7. Doi: 10.4018/978-4666-1833-6.ch012

[82] Collobert R, Weston J, Bottou L, et al. 2011. Natural language processing (almost) from scratch. Journal of Machine Learning; 12: 2493-537

[83] Huang W, Nakamori Y, Wang SY. 2005. Forecasting stock market movement direction with support vector machine. Computer \& Operations Research; 32: 2513-22

[84] Wang S, Summers RM. 2012. Machine learning and radiology. Med Image Anal; 16(5): 933-51

[85] Hassanpour S, Langlotz CP. 2015. Information extraction from multi-institutional radiology reports. Artif Intell Med; 66: 29-39

[86] Douglas PK, Harris S, Yuille Aet al. 2011. Performance comparison of machine learning algorithms and number of independent components using in fMRI decoding of belief vs. disbelief. Neuroimage; 56(2): 544-53

[87] Cunningham JP, Ghahramani Z. 2015. Linear dimensionality reduction: survey, insights, and generalizations. Journal of Machine Learning Research; 16: 2859-900

[88] Rodda SL, Tyldesley S, Morris WJ. 2015. Toxicity outcomes in ASCENDERT: a multicenter randomized trial of dose-escalation trial for prostate cancer. Int J 
Radiat Oncol Biol Phys; 93(3): S121 [Abstract]

[89] Keyes M, Crook J, Morris WJ, et al. 2013. Canadian prostate brachytherapy in 2012. Can Urol Assoc; 7: 51-8

[90] Dickinson PD, Malik J, Mandall P, et al. 2013. Five-year outcomes after iodine-125 seed brachytherapy for low-risk prostate cancer at three cancer centres in the UK. BJU Int; 113(5): 748-53

[91] Rodrigues G, Yao X, Loblaw DA, et al. 2013. Low-dose rate brachytherapy for patients with low- or intermediate-risk prostate cancer: a systematic review. Can Urol Assoc J; 7(11-12): 463-70

[92] Cozzarini C. 2011. Low-dose-rate brachytherapy, radical prostatectomy, or external-beam radiation therapy for localized prostate carcinoma: the growing dilemma. Eur Urol; 60: 894-6

[93] Smith GD, Pickles T, Crook J, et al. 2014. Brachytherapy improves biochemical failure-free survival in low-and intermediate-risk prostate cancer compared with conventionally fractionated external beam radiation therapy: a propensity score matched analysis. Int J Radiat Oncol Biol Phys; 91(3): 506-16

[94] Zelefsky MJ, Chou JF, Pei X, et al. 2012. Predicting biochemical tumor control after brachytherapy for clinically localized prostate cancer: the Memorial Sloan-Kettering Cancer Center experience. Brachytherapy; 11: 245-9

[95] Kupelian PA, Potters L, Khuntia D, et al. 2004. Radical prostatectomy, external beam radiotherapy $<72$ Gy, external beam radiotherapy $>72$ Gy, permanent seed implantation, or combined seeds / external beam radiotherapy for stage T1-T2 prostate cancer. Int J Radiat Oncol Biol Phys; 58(1): 25-33

[96] Lo AC, Morris WJ, Pickles T, et al. 2015. Patterns of recurrence after lowdose-rate prostate brachytherapy: a population-based study of 2223 consecutive lowand intermediate-risk patients. Int J Radiat Oncol Biol Phys; 91(4): 745-51

[97] Al-Qaisieh B, Witteveen T, Carey B, et al. 2009. Correlation between preand postimplant dosimetry for iodine-125 seed implants for localized prostate cancer. Int J Radiat Oncol Biol Phys; 75(2): 626-30

[98] Lacy JM, Wilson WA, Bole R, et al. 2016. Salvage brachytherapy for biochemically recurrent prostate cancer following primary brachytherapy. Prostate Cancer; [epub], doi: 10.1155/2016/9561494

[99] Betts JM, Mears C, Reynolds HM, et al. 2015. Optimised robust treatment plans for prostate cancer focal brachytherapy. Procedia Computer Science; 51: 914-23

[100] Ertekin S, Huang J, Giles CL. 2007. Active learning for class imbalance problem. SIGIR: 823-4. Doi: 10.1145/1277741.1277927 
[101] Gregory KJ, Pattison JE, Bibbo G. 2015. Measurement uncertainty analysis of low-dose-rate prostate seed brachytherapy: post-implant dosimetry. Australasian Physical \& Engineering Sciences in Medicine; 38(1): 71-81

[102] Wang X, Paliwal KK. 2003. Feature extraction and dimensionality reduction algorithms and their applications in vowel recognition. Pattern Recognition; 36 : 2429-39

[103] Holmes DR, Davis BJ, Goulet CC, et al. 2014. Shape analysis of the prostate: establishing imaging specifications for the design of a transurethral imaging device for prostate brachytherapy guidance. Brachytherapy; 13: 465-70

[104] O'Mara D, Owens R. 1996. Measuring bilateral symmetry in digital images. IEEE TENCON; 1: 151-6. Doi: 10.1109/TENCON.1996.608740

[105] Park SB, Kim SK, Choi SH, et al. 2000. Prostate volume measurement by TRUS using heights obtained by transaxial and midsagittal scanning: comparison with specimen volume following radical prostatectomy. Korean J Radiol; 1: 110-113

[106] Cheadle C, Vawter MP, Freed WJ, et al. 2003. Analysis of microarray data using z score transformation. J Mol Diagn; 5(2): 73-81

[107] Xin X, Ma Z, Katsaggelos AK. 2013. Spectral approximation to point set similarity metric. ICMEW/IEEE Proc: 1-4. Doi: 10.1109/ICMEW.2013.6618283

[108] Keogh E, Celly B, Ratanamahatana CA, et al. 2003. A novel technique for indexing video surveillance data. Proc IWVS: 98-106

[109] Chechik G, Sharma V, Shalit U, et al. 2010. Large scale online learning of image similarity through ranking. Journal of Machine Learning Research; 11: 1109-35

[110] Krstajic D, Buturovic LJ, Leahy DE, et al. 2014. Cross-validation pitfalls when selecting and assessing regression and classification models. J Cheminform; 6(1): 10. Doi: $10.1186 / 1758-2946-6-10$

[111] Davis BJ, Horwitz EM, Lee WR, et al. 2012. American Brachytherapy Society consensus guidelines for transrectal ultrasound-guided permanent prostate brachytherapy. Brachytherapy; 11(1): 6-19

[112] Lake BM, Salakhutdinov R, Tenenbaum JB. 2015. Human-level concept learning through probabilistic program induction. Science; 350(6266): 1332-8

[113] Ganjisaffar Y, Debeauvais T, Javanmardi S, et al. 2011. Distributed tuning of machine learning algorithms using MapReduce clusters. LDMTA; 2. Doi: $10.1145 / 2002945.2002947$

[114] Srivastava N, Hinton G, Krizhevsky A, et al. 2014. Dropout: A simple 
way to prevent neural networks from overfitting. Journal of Machine Learning Research; 15: 1929-58

[115] Skowronek J. 2013. Low-dose-rate or high-dose-rate brachytherapy in treatment of prostate cancer - between options. J Contemp Brachytherapy; 5(1): 33-41

[116] Agarwal S, Mozafari B, Panda A, et al. 2013. BlinkDB: Queries with bounded errors and bounded response times on very large data. Eurosys; 13: 29-42

[117] Gao L, Wang M, Wang S, et al. 2004. Expressing and optimizing similarity-based queries in SQL. Conceptual Modeling; 3288: 464-78

[118] Sparks R, Madabhushi A. 2011. Content-based image retrieval utilizing explicit shape descriptors: applications to breast MRI and prostate histopathology. Proc of SPIE; 7962

[119] Mishra N, Petrovic S, Sundary S. 2011. A self-adaptive case-based reasoning system for dose planning in prostate cancer radiotherapy. Med Phys; 38: 6528-37

[120] Susil RC, Camphausen K, Choyke P, et al. 2004. System for prostate brachytherapy and biopsy in a standard 1.5T MRI scanner. Magn Reson Med; 52(3): 683-7

[121] Menard C, Susil RC, Choyke P, et al. 2004. MRI-guided HDR prostate brachytherapy in standard 1.5T scanner. Int J Radiat Oncol Biol Phys; 59(5): 1414-23

[122] Arevalo J, Gonzalez FA, Ramos-Pollan, et al. 2016. Representation learning for mammography mass lesion classification with convolutional neural networks. Comput Methods Programs Biomed; 127: 248-57

[123] Ghose S, Mitra J, Oliver A, et al. 2012. A supervised learning framework for automatic segmentation in trans rectal ultrasound images. Advanced Concepts for Intelligent Vision Systems; 7517: 190-200

[124] Bottou L. 2010. Large-scale machine learning with stochastic gradient descent. Proceedings of COMPSTAT'2010. P 177-86

[125] Maclaurin D, Duvenaud D, Adams RP. 2015. Gradient-based hyperparameter optimization through reversible learning. Proceedings of 32nd International Conference on Machine Learning; 37.

[126] Nath S, Chen Z, Yue N, et al. 2000. Dosimetric effects of needle divergence in prostate seed implant using I and Pd radioactive seeds. Med Phys; 27: 1058-66

[127] Beaulieu L, Archambault L, Aubin S, et al. 2004. The robustness of dose distributions to displacement and migration of $125 \mathrm{I}$ permanent seed implants over a wide range of seed number, activity, and designs. Int J Radiat Oncol Biol Phys; 58(4): 1298-308 
[128] Yamada Y, Rogers L, Demanes DJ, et al. 2012. American Brachytherapy Society consensus guidelines for high-dose-rate prostate brachytherapy. Brachytherapy; 11(1): 20-32

[129] Doi K. 2005. Current status and future potential of computer-aided diagnosis in medical imaging. Br J Radiol; 78 Spec No 1: S3-19

[130] Jayasurya K, Fung G, Yu S, et al. 2010. Comparison of Bayesian network and support vector machine models for two-year survival prediction in lung cancer patients treated with radiotherapy. Med Phys; 37(4): 1401-7

[131] Lambin P, Roelofs E, Reymen B, et al. 2013. Rapid learning health care in oncology âĂŞ an approach towards decision support systems enabling customized radiotherapy. Radiother Oncol; 109(1): 159-64

[132] Li W et al. 2012. Learning image context for segmentation of the prostate in CT-guided radiotherapy. Phys Med Biol; 57(5): 1283-308

[133] Petrovic S, Mishra N, Sundar S, et al. 2011. A novel case based reasoning approach to radiotherapy planning. Expert Syst Appl; 38: 1759-69

[134] El Naqa I, Li R, Murphy MJ. 2015. Machine Learning in Radiation Oncology. Springer Int. Publishing. Switzerland. Doi: 10.1007/978-3-319-18305-3 